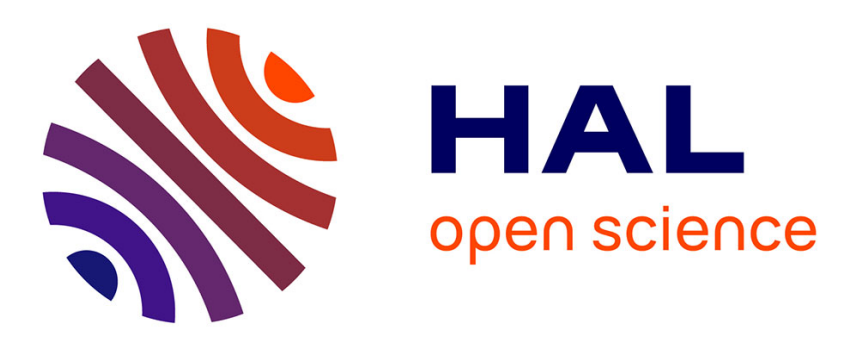

\title{
Robust hierarchic control for a population dynamics model with missing birth rate
}

\author{
Gisèle Mophou, M Kéré, L L Djomegne Njoukoue
}

\section{To cite this version:}

Gisèle Mophou, M Kéré, L L Djomegne Njoukoue. Robust hierarchic control for a population dynamics model with missing birth rate. Mathematics of Control, Signals, and Systems, 2020. hal-02552534

HAL Id: hal-02552534

https://hal.science/hal-02552534

Submitted on 23 Apr 2020

HAL is a multi-disciplinary open access archive for the deposit and dissemination of scientific research documents, whether they are published or not. The documents may come from teaching and research institutions in France or abroad, or from public or private research centers.
L'archive ouverte pluridisciplinaire HAL, est destinée au dépôt et à la diffusion de documents scientifiques de niveau recherche, publiés ou non, émanant des établissements d'enseignement et de recherche français ou étrangers, des laboratoires publics ou privés. 


\title{
Robust hierarchic control for a population dynamics model with missing birth rate
}

\author{
G. MOPHOU ${ }^{*}$ M. KÉRÉ ${ }^{\dagger}$ L. L. DJOMEGNE NJOUKOUE ${ }^{\ddagger}$
}

August 24, 2019

\begin{abstract}
In this paper we study the hierarchic control problem for a linear system of a population dynamics model with unknown birth rate. Using the notion of low regret control and an observability inequality of Carleman type, we show that there exist two controls such that, the first control called follower solves an optimal control problem which consist in bringing the state of the linear system to desired state, and the second one named leader is supposed to lead the population to extinction at final time.
\end{abstract}

Mathematics Subject Classification. 49J20,92D25,93B05; 93C41.

Key-words : Population dynamics, Carleman inequality, incomplete data, optimal control, low-regret control, controllability, Euler-Lagrange formula.

\section{Introduction}

We consider a population with age dependence and spatial structure, and we assume that the population lives in a bounded domain $\Omega \subset \mathbb{R}^{n}, n \in \mathbb{N}^{*}$, with boundary $\Gamma$ of class $C^{2}$. Let $\tilde{y}=\tilde{y}(t, a, x)$ be the distribution of individuals of age $a \geq 0$, at time $t \geq 0$ and location $x \in \Omega$. Let also $A>0$, be the life expectancy of an individual and the final time $T$, be a positive constant. Let $\omega$ and $\mathcal{O}$ be nonempty subsets of $\Omega$ with $\omega \varsubsetneqq \mathcal{O}$. Set $U=(0, T) \times(0, A)$, $Q=U \times \Omega, \Sigma=U \times \Gamma, Q_{A}=(0, A) \times \Omega, Q_{T}=(0, T) \times \Omega, Q_{\omega}=U \times \omega$ and $Q_{\mathcal{O}}=U \times \mathcal{O}$. We denote by $\tilde{\mu}=\tilde{\mu}(t, a, x) \geq 0$, the natural death rate of

* African Institute for Mathematical Sciences (AIMS), P.O. Box 608, Limbe Crystal Gardens, South West Region, Cameroon-Laboratoire LAMIA, Université des Antilles, Campus Fouillole, 97159 Pointe-à-Pitre Guadeloupe (FWI)- Laboratoire MAINEGE, Université Ouaga 3S, O6 BP 10347 Ouagadougou 06, Burkina Faso, email : gisele.mophou@univantilles.fr gisele.mophou@aims-cameroon.org

${ }^{\dagger}$ Institut des Sciences (IDS), Departement de mathematics, 01 BP 1757 Ouaga 01, Burkina Faso, email : moumik3000@gmail.com

$\ddagger$ University of Dschang, BP 67 Dschang, Cameroon, West region, email : landry.djomegne@yahoo.fr 
individuals of age $a$ at time $t$ and location $x$. We consider the following linear system of population dynamics model:

$$
\left\{\begin{aligned}
\frac{\partial \tilde{y}}{\partial t}+\frac{\partial \tilde{y}}{\partial a}-\Delta \tilde{y}+\tilde{\mu} \tilde{y} & =\tilde{k} \chi_{\omega}+\tilde{v} \chi_{\mathcal{O}} & & \text { in } Q, \\
\tilde{y} & =0 & & \text { on } \Sigma, \\
\tilde{y}(0, ., .) & =0 & & \text { in } Q_{A}, \\
\tilde{y}(., 0, .) & =\tilde{g} & & \text { in } \quad Q_{T},
\end{aligned}\right.
$$

where the controls $\tilde{v}$ and $\tilde{k}$ belong to $L^{2}(Q), \chi_{\omega}$ and $\chi_{\mathcal{O}}$ denote the characteristic functions of the control sets $\omega$ and $\mathcal{O}$ respectively, $\tilde{g}$ belongs to $L^{2}\left(Q_{T}\right)$ is unknown and represents the distribution of newborn individuals at time $t$ and location $x$. We make the following assumptions:

$$
(H):\left\{\begin{array}{c}
\tilde{\mu}(t, a, x)=\tilde{\mu}_{0}(a)+\tilde{\mu}_{1}(t, a, x) \text { in } Q \\
\tilde{\mu}_{1} \in L^{\infty}(Q) ; \tilde{\mu}_{1}(t, a, x) \geq 0 \text { for }(t, a, x) \in Q \\
\tilde{\mu}(t, a, x) \geq 0 \text { for }(t, a, x) \in Q \\
\tilde{\mu}_{0} \geq 0, \tilde{\mu}_{0} \in L_{l o c}^{1}(0, A), \lim _{a \rightarrow A} \int_{0}^{a} \tilde{\mu}_{0}(s) d s=+\infty
\end{array}\right.
$$

The fourth assumption in $(H)$ means that all individual dies before the age $A$. For more literature on the signification of assumption $(H)$ as well as on the population dynamics equations, we refer to $[1,2,3]$ and the reference therein.

The Stackelberg leadership model is a multiple-objective optimization approach proposed by H. Von Stackelberg in [6]. This model involves two companies (controls) which compete on the market of the same product. The first(leader) to act must integrate the reaction of the other firm (followers) in the choices it makes in the amount of product that it decides to put on the market. There are several works in the literature dealing with Stackelberg strategy for distributed systems. J. L. Lions [7] used the Stackelberg strategy on a system governed by a parabolic equation subjected to two controls. The follower acts on the system in order to bring the state not far from a desired state while the leader has to steer the state at final time to a small neighborhood of a given state. O. Nakoulima [8] used this concept control for a backward heat equation involving two controls to determine: one of null controllability type with constraint on the control, called follower, and the other of optimal control type, called leader. The results were achieved by means of a Carleman inequality adapted to the constraint. In $[9,10]$, M. Mercan revisited the notion of controllability in the sense of Stackelberg given by O. Nakoulima [8] by choosing the follower of minimal norm. This new notion is then applied by O. Nakoulima et al. in [11] on the controllability of a two-stroke problem with constraint on the states. The results were obtained by means of Carleman inequality adapted to the constraints. Recently G. Mophou et al. [12] considered the Stackelberg problem for coupled parabolic equations with a finite number of constraints on one of the states. The first control was supposed to bring the solution of the coupled system subjected to a finite number of constraints at rest at time $\mathrm{T}$ while the second expresses that the states do not move too far from a given state. 
In this paper we are interesting in the Robust hierarchic control for the population dynamics equation with missing birth rate:

$$
\left\{\begin{aligned}
\frac{\partial y}{\partial t}+\frac{\partial y}{\partial a}-\Delta y+\mu y & =k \chi_{\omega}+v \chi_{\mathcal{O}} & & \text { in } Q, \\
y & =0 & & \text { on } \Sigma, \\
y(0, ., .) & =0 & & \text { in } Q_{A}, \\
y(., 0, .) & =g & & \text { in } Q_{T},
\end{aligned}\right.
$$

where

$$
\begin{array}{r}
y(t, a, x)=\wp(t, a) \tilde{y}(t, a, x), k(t, a, x)=\wp(t, a) \tilde{k}(t, a, x), \\
v(t, a, x)=\wp(t, a) \tilde{v}(t, a, x), g(t, x)=\wp(t, 0) \tilde{g}(t, x),
\end{array}
$$

with

$$
\wp(t, a)=\exp \left(-r_{0} t+\int_{0}^{a} \tilde{\mu}_{0}(s) d s\right),
$$

and $\mu=\tilde{\mu}_{1}+r_{0}$. The nonnegative constant $r_{0} \geq 1 / 2$.

In view of this change of variables, assumptions $(H)$, and the fact that $k \chi_{\omega}, v \chi_{\mathcal{O}} \in L^{2}(Q)$ and $g \in L^{2}\left(Q_{T}\right)$, we know that problem (1) has a unique solution $y=y(k ; v, g)$ in $L^{2}\left(U, H_{0}^{1}(\Omega)\right)$ and $\frac{\partial y}{\partial t}+\frac{\partial y}{\partial a} \in L^{2}\left(U ; H^{-1}(\Omega)\right)$. (See $[4,5])$. Moreover the following estimation holds:

$$
\begin{array}{r}
\|y(T, ., .)\|_{L^{2}\left(Q_{A}\right)}^{2}+\|y(., A, .)\|_{L^{2}\left(Q_{T}\right)}^{2} \leq 4\left(\|g\|_{L^{2}\left(Q_{T}\right)}^{2}+\|k\|_{L^{2}\left(Q_{\omega}\right)}^{2}+\|v\|_{L^{2}\left(Q_{\mathcal{O}}\right)}^{2}\right), \\
\|y\|_{L^{2}\left(U, H_{0}^{1}(\Omega)\right)}^{2} \leq 4\left(\|g\|_{L^{2}\left(Q_{T}\right)}^{2}+\|k\|_{L^{2}\left(Q_{\omega}\right)}^{2}+\|v\|_{L^{2}\left(Q_{\mathcal{O}}\right)}^{2}\right) .
\end{array}
$$

One comes across model (1) while describing the dynamic of some invasive species( fish) in a lake for instance. This kind of species can come from everywhere including pollution. In this paper, we assume that we have some measures, some information but we don't have at our disposal the distribution of newborns, which is here expressed by the unknown variable $g$. So, we want to drive the distribution of the species to zero with appropriate control acting on a sub-domain of the lake, trying meanwhile to keep the distribution of the species to the desired state with another control acting in another sub-domain of the lake. We thus consider the following problems.

Problem 1 Let $k \in L^{2}\left(Q_{\omega}\right)$. For any $\gamma>0$, find the best control $\hat{v}^{\gamma}=\hat{v}^{\gamma}(k) \in$ $L^{2}\left(Q_{\mathcal{O}}\right)$ solution of

$$
\inf _{v \in L^{2}\left(Q_{\mathcal{O}}\right)} \sup _{g \in L^{2}\left(Q_{T}\right)}\left[J(k ; v, g)-J(0 ; 0, g)-\gamma\|g\|_{L^{2}\left(Q_{T}\right)}^{2}\right],
$$

where

$$
J(k ; v, g)=\left\|y(k ; v, g)-z_{d}\right\|_{L^{2}(Q)}^{2}+N\|v\|_{L^{2}\left(Q_{\mathcal{O}}\right)}^{2},
$$

with $z_{d} \in L^{2}(Q)$ and $N>0$. 
Problem 2 Let $\hat{v}^{\gamma}(k)$ be the control obtain in the first objective and $\hat{y}^{\gamma}=$ $y\left(t, a, x ; k ; \hat{v}^{\gamma}(k)\right)$ be the associated state. Find the control $k \in L^{2}\left(Q_{\omega}\right)$ such that

$$
\hat{y}^{\gamma}(T)=y\left(T, a, x ; k ; \hat{v}^{\gamma}\right)=0, \quad(0, A) \times \Omega .
$$

The problem consider in the first problem is an optimization associated to the problem with missing birth rate. Such problem has been study by B. Jacob et al. in [13] for a linear population dynamic equation involving with missing initial distribution of individuals of age $a \in(0, A)$. To solve this problem the author used the notion of No-regret and Low-regret control introduced by J. L. Lions [14] and obtained a singular optimality conditions which characterize the No-regret control. More precisely, they consider for any $\gamma>0$ the optimization problem:

$$
\inf _{v \in L^{2}\left(Q_{\mathcal{O}}\right)} \sup _{g \in L^{2}\left(Q_{T}\right)}\left[J(v, g)-J(0, g)-\gamma\|g\|_{L^{2}\left(Q_{T}\right)}^{2}\right],
$$

called Low-regret problem. Then, they proved that the Low regret control $u_{\gamma}$ converges towards the solution of the No-regret control problem:

$$
\inf _{v \in L^{2}\left(Q_{\mathcal{O}}\right)} \sup _{g \in L^{2}\left(Q_{T}\right)}[J(v, g)-J(0, g)],
$$

that they characterized assuming that the control acts on the whole domain $(\mathcal{O}=\Omega)$. The second problem is a null controllability problem associate to a population dynamics equations. Actually, after solving the first problem, the second consists in solving a null controllability problem associated to a cascade of two stroke equations. Controllability problems for an age and space structured population dynamics model have been studied by several authors. B. Ainseba proved in [15] the exact and approximate controllability for linear population dynamics problem structured in age and space. In [16], S. Anita et al. showed that if the initial distribution is small enough, one can find a control which leads to extinction of the population. Using Kakutani fixed point theorem, B. M. Iannelli et al. established a null controllability result for nonlinear population dynamics model in [25]. In [17] S. Sawadogo et al. gave a null controllability result for population dynamics model with constraints on the state when the age of the population belongs to $(\gamma, A)$ for any $\gamma>0$. In [18] M. Langlais et al. studied a population dynamics control problem with age dependence and spatial structure. In [19], Echarroudi et al. study the null controllability of a linear model with degenerate diffusion in population dynamics. The results is achieved by means of an appropriate observability inequality and a fixed point technique. In [20] M. Tucsnak et al studied the null controllability of a linear population dynamics model with a control localized in the space variable as well as with respect to the ages. They proved in the one hand that the age interval in which the control needs to be active can be arbitrarily small, and on the other hand that, that the whole population can be steered into zero in an uniform time. In [29], N. Hegoburu et al. considered a non linear model system describing age structured population dynamics, where the birth and the mortality rates are nonlinear functions of the population size. They gave sharp 
conditions subject to the age range and the control time horizon to get the null controllability of the nonlinear controlled population dynamics when the control is active on some age range. For more literature on the controllability fo population dynamics equation we refer to $[21,22,23,24, ?, 25]$ and the reference therein.

In this paper, we propose the Stackelberg control problem with missing data using the notion of Low-regret control for the follower, and appropriate Carleman for the leader. More precisely, we prove the following results.

Theorem 1 Let $\Omega$ be a bounded subset of $\mathbb{R}^{n}, n \geq 1$ with boundary $\Gamma$ of class $C^{2}$. Let $\omega$ and $\mathcal{O}$ be nonempty subsets of $\Omega$ with $\omega \varsubsetneqq \mathcal{O}$. Let also $k \in L^{2}\left(Q_{\mathcal{O}}\right)$ and $\gamma>0$. Then there exists $\left(S^{\gamma}, p^{\gamma}, q^{\gamma}\right)$ such that the optimization problem (5) has a unique solution $v^{\gamma}=v^{\gamma}(k) \in L^{2}\left(Q_{\mathcal{O}}\right)$ which is characterized by the following optimality system:

$$
\begin{aligned}
& \left\{\begin{aligned}
L y^{\gamma} & =v^{\gamma} \chi_{\mathcal{O}}+k \chi_{\omega} & & \text { in } Q, \\
y^{\gamma} & =0 & & \text { on } \Sigma, \\
y^{\gamma}(0, . . .) & =0 & & \text { in } Q_{A}, \\
y^{\gamma}(., 0, .) & =0 & & \text { in } Q_{T},
\end{aligned}\right. \\
& \begin{array}{c}
\left\{\begin{array}{rlll}
L^{*} S^{\gamma}=y^{\gamma} \quad \text { in } \quad Q, \\
S^{\gamma}=0 \quad \text { on } \Sigma, \\
S^{\gamma}(T, ., .)=0 \quad \text { in } Q_{A}, \\
S^{\gamma}(., A, .)=0 \quad \text { in } Q_{T}, \\
L p^{\gamma}=0 & & \text { in } Q, \\
p^{\gamma}=0 & \text { on } \Sigma, \\
p^{\gamma}(0, ., .)=0 & \text { in } Q_{A}, \\
p^{\gamma}(., 0, .)=\frac{1}{\sqrt{\gamma}} S^{\gamma}(., 0, .) & \text { in } Q_{T},
\end{array}\right.
\end{array}
\end{aligned}
$$

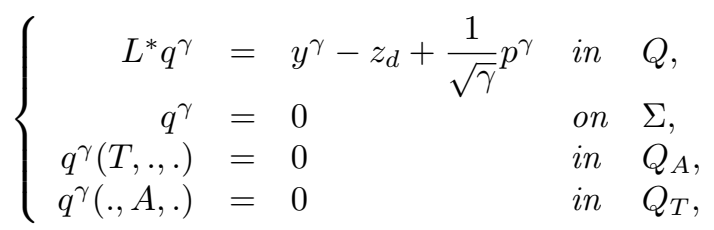

and

$$
v^{\gamma}=-\frac{q^{\gamma}}{N} \text { in } Q_{\mathcal{O}}
$$

with the operators $L$ and $L^{*}$ defined as

$$
\begin{aligned}
L & =\frac{\partial}{\partial t}+\frac{\partial}{\partial a}-\Delta+\mu I, \\
L^{*} & =-\frac{\partial}{\partial t}-\frac{\partial}{\partial a}-\Delta+\mu I
\end{aligned}
$$

and I the operator identity.

Moreover there exists a constant $C>0$ independent of $\gamma$ such that

$$
\left\|v^{\gamma}\right\|_{L^{2}\left(Q_{\mathcal{O}}\right)} \leq C\left(\left\|z_{d}\right\|_{L^{2}(Q)}+\|k\|_{L^{2}\left(Q_{\omega}\right)}\right) .
$$


Theorem 2 Assume that the assumptions of Theorem 1 hold. Then there exists a positive real weight function $\kappa$ (a precise definition of $\kappa$ will be given later on) such that, for any function $z_{d} \in L^{2}(Q)$ with $\frac{1}{\kappa} z_{d} \in L^{2}(Q)$, then there exists a unique control $\hat{k}^{\gamma} \in L^{2}\left(Q_{\omega}\right)$ such that $\left(\hat{k}^{\gamma}, \hat{y}^{\gamma}, \hat{S}^{\gamma}, \hat{p}^{\gamma}, \hat{q}^{\gamma}\right)$ is the solution of the null controllability problem (8)-(7). Moreover

$$
\hat{k}^{\gamma}=\hat{\rho}^{\gamma} \chi_{\omega}
$$

where $\hat{\rho}^{\gamma}, \hat{\phi}^{\gamma}, \hat{\phi}^{\gamma}$ and $\hat{\zeta}^{\gamma}$ are solutions of

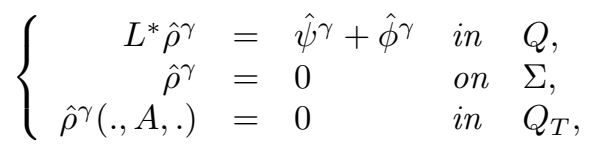

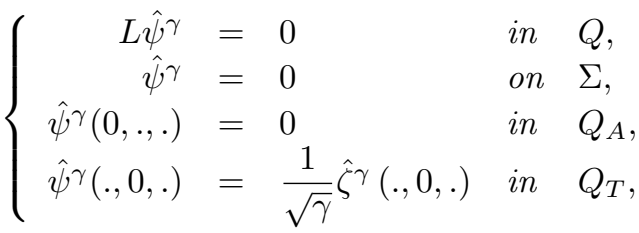

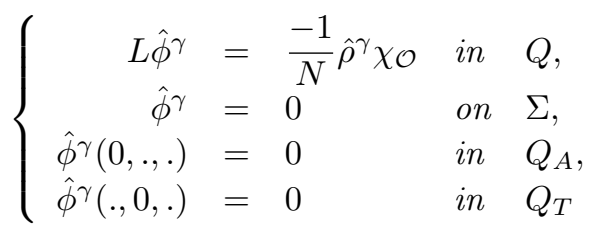

and

$$
\left\{\begin{array}{rlrl}
L^{*} \hat{\zeta}^{\gamma} & =\frac{1}{\sqrt{\gamma}} \hat{\phi}^{\gamma} & \text { in } \quad & Q, \\
\hat{\zeta}^{\gamma} & =0 & & \text { on } \quad \Sigma, \\
\hat{\zeta}^{\gamma}(T, . . .) & =0 & \text { in } & Q_{A}, \\
\hat{\zeta}^{\gamma}(., A, .) & =0 & \text { in } \quad Q_{T} .
\end{array}\right.
$$

In addition, there exists a constant $C>0$ independent of $\gamma$ such that

$$
\left\|\hat{k}^{\gamma}\right\|_{L^{2}\left(Q_{\omega}\right)} \leq C\left\|\frac{1}{\kappa} \varrho z_{d}\right\|_{L^{2}(Q)} .
$$

The rest of this paper is organized as follows. Section 2 is devoted to the proof of Theorem 1. In Section 3, we establish an appropriate inequality of Carleman type and give the proof of the Theorem 2. Concluding remarks are given in Section 4.

\section{Study of optimization problem}

In this section, $k$ is fixed and we are concerned with optimization (5):

$$
\inf _{v \in L^{2}\left(Q_{\mathcal{O}}\right)} \sup _{g \in L^{2}\left(Q_{T}\right)}\left[J(v, g)-J(0, g)-\gamma\|g\|_{L^{2}\left(Q_{T}\right)}^{2}\right],
$$


where $J$ is given by (6).

Let $y=y(k ; v, g)=y(t, a, x ; k ; v, g)$ be the solution of $(2)$. Then

$$
y(k ; v, g)=y(k ; v, 0)+y(0 ; 0, g),
$$

where $y(k ; v, 0)$ and $y(0 ; 0, g)$ are respectively solutions of

$$
\left\{\begin{aligned}
L y(k ; v, 0) & =v \chi_{\mathcal{O}}+k \chi_{\omega} & & \text { in } Q, \\
y(k ; v, 0) & =0 & & \text { on } \Sigma, \\
y(0, ., . ; k ; v, 0) & =0 & & \text { in } Q_{A} \\
y(., 0, . ; k ; v, 0) & =0 & & \text { in } Q_{T},
\end{aligned}\right.
$$

and

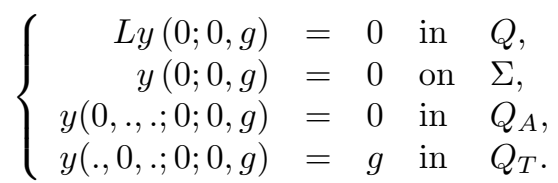

Since $g \in L^{2}\left(Q_{T}\right), v \in L^{2}(Q)$ and $k \in L^{2}(Q)$, we know (see e.g. [4]) that the functions $y(k ; v, 0)$ and $y(0 ; 0, g)$ belong to $L^{2}\left(U ; H_{0}^{1}(\Omega)\right)$. Using this decomposition of the state equation, we have after some calculations

$$
\begin{aligned}
J(k ; v, g)= & J(k ; v, 0)+J(0 ; 0, g)-\left\|z_{d}\right\|_{L^{2}(Q)}^{2} \\
& +2 \int_{Q} y(k ; v, 0) y(0 ; 0, g) d t d a d x
\end{aligned}
$$

where

$$
\begin{aligned}
& J(k ; v, 0)=\left\|y(k ; v, 0)-z_{d}\right\|_{L^{2}(Q)}^{2}+N\|v\|_{L^{2}\left(Q_{\mathcal{O}}\right)}^{2} \\
& J(0 ; 0, g)=\left\|y(0 ; 0, g)-z_{d}\right\|_{L^{2}(Q)}^{2} .
\end{aligned}
$$

Therefore

$$
\begin{aligned}
J(k ; v, g)-J(0 ; 0, g) & =J(k ; v, 0)-\left\|z_{d}\right\|_{L^{2}(Q)}^{2} \\
& +2 \int_{Q} y(k ; v, 0) y(0 ; 0, g) d t d a d x .
\end{aligned}
$$

Consider now the adjoint state $S(k ; v)=S(t, a, x ; k ; v) \in L^{2}\left(U ; H_{0}^{1}(\Omega)\right)$, solution of

$$
\left\{\begin{aligned}
L^{*} S(k ; v) & =y(k ; v, 0) & & \text { in } Q, \\
S(k ; v) & =0 & & \text { on } \Sigma, \\
S(T, ., .) & =0 & & \text { in } Q_{A}, \\
S(., A, .) & =0 & & \text { in } Q_{T} .
\end{aligned}\right.
$$

Multiplying the first equation in $(23)$ by $y(0 ; 0, g)$ and integrating by parts over $Q$, we obtain that

$$
\int_{Q} y(k ; v, 0) y(0 ; 0, g) d t d a d x=\int_{Q_{T}} S(t, 0, x ; k ; v) g d t d x .
$$


Combining (22) and (24), we deduce that

$$
J(k ; v, g)-J(0 ; 0, g)=J(k ; v, 0)-\left\|z_{d}\right\|_{L^{2}(Q)}^{2}+2 \int_{Q_{T}} S(t, 0, x ; k ; v) g d t d x
$$

On the other hand, for any $\gamma>0$ we have

$$
\begin{aligned}
& \sup _{g \in L^{2}\left(Q_{T}\right)}\left\{J(k ; v, g)-J(0 ; 0, g)-\gamma\|g\|_{L^{2}\left(Q_{T}\right)}^{2}\right\}=J(k ; v, 0)-\left\|z_{d}\right\|_{L^{2}(Q)}^{2}+ \\
& 2 \sup _{g \in L^{2}\left(Q_{T}\right)}\left\{\int_{Q_{T}} S(t, 0, x ; k ; v) g d t d x-\frac{\gamma}{2}\|g\|_{L^{2}\left(Q_{T}\right)}^{2}\right\},
\end{aligned}
$$

which by Legendre-Fenchel transform gives

$$
\sup _{g \in L^{2}\left(Q_{T}\right)}\left\{J(k ; v, g)-J(0 ; 0, g)-\gamma\|g\|_{L^{2}\left(Q_{T}\right)}^{2}\right\}=J^{\gamma}(k ; v),
$$

where

$$
J^{\gamma}(k ; v)=J(k ; v, 0)-\left\|z_{d}\right\|_{L^{2}(Q)}^{2}+\frac{1}{\gamma}\|S(., 0, . ; k ; v)\|_{L^{2}\left(Q_{T}\right)}^{2} .
$$

Hence the Low-regret control problem (5) is equivalent to the following optimal control problem: Let $k \in L^{2}\left(Q_{\omega}\right)$. For any $\gamma>0$, find $v^{\gamma}=v^{\gamma}(k) \in L^{2}\left(Q_{\mathcal{O}}\right)$ such that

$$
J^{\gamma}\left(k ; v^{\gamma}\right)=\inf _{v \in L^{2}\left(Q_{\mathcal{O}}\right)} J^{\gamma}(k ; v) .
$$

Remark 3 If we consider in problem (5), with $\gamma=0$ :

$$
\inf _{v \in L^{2}\left(Q_{\mathcal{O}}\right)} \sup _{g \in L^{2}\left(Q_{T}\right)}[J(k ; v, g)-J(0 ; 0, g)],
$$

then we deal with the No-regret control problem. Therefore in view of (25), the No-regret control $\hat{v}$ belongs to the set

$$
\mathcal{U}=\left\{v \in L^{2}\left(Q_{\mathcal{O}}\right) \text { such that } \int_{Q_{T}} S(t, 0, x ; k ; v) g d t d x=0 \quad \forall g \in L^{2}\left(Q_{T}\right)\right\} .
$$

\subsection{Proof of Theorem 1}

Since $J^{\gamma}(k ; v) \geq-\left\|z_{d}\right\|_{L^{2}(Q)}^{2}$, we can prove using minimizing sequence and standard arguments that there exists a unique Low-regret control $v^{\gamma}$ solution to problem (28).

To characterize the optimal control $v^{\gamma}$, we make use of the Euler-Lagrange optimality conditions:

$$
\lim _{\lambda \rightarrow 0} \frac{J^{\gamma}\left(k ; v^{\gamma}+\lambda v\right)-J^{\gamma}\left(k ; v^{\gamma}\right)}{\lambda}=0, \quad \forall v \in L^{2}\left(Q_{\mathcal{O}}\right) .
$$


After a short calculation, (31) gives,

$$
\begin{aligned}
0 & =\int_{Q} \bar{y}\left\{y\left(k ; v^{\gamma}, 0\right)-z_{d}\right\} d t d a d x+N\left(v^{\gamma}, v\right)_{L^{2}\left(Q_{\mathcal{O}}\right)} \\
& +\frac{1}{\gamma} \int_{Q_{T}} \bar{S}(t, 0, x ; k ; v) S\left(t, 0, x ; k ; v^{\gamma}\right) d t d x, \quad \forall v \in L^{2}\left(Q_{\mathcal{O}}\right)
\end{aligned}
$$

where $\bar{y}=\bar{y}(t, a, x ; 0 ; v, 0)$ and $\bar{S}(0 ; v)=\bar{S}(t, a, x ; 0 ; v)$ are respectively solution of

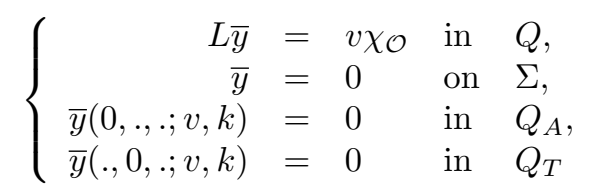

and

$$
\left\{\begin{array}{rllll}
L^{*} \bar{S} & = & \bar{y} & \text { in } & Q \\
\bar{S} & = & 0 & \text { on } \quad \Sigma \\
\bar{S}(T, . ., ; v) & = & 0 & \text { in } \quad Q_{A} \\
\bar{S}(., A, . ; v) & = & 0 & \text { in } \quad Q_{T} .
\end{array}\right.
$$

To interpret (32), we use $p^{\gamma}$ and $q^{\gamma}$ respectively solutions of (10) and (11). So if we multiply $(33)_{1}$ by $q^{\gamma}$ and $(34)_{1}$ by $\frac{1}{\sqrt{\gamma}} p^{\gamma}$ and integrate by parts over $Q$ we respectively obtain that

$$
\int_{Q} \bar{y}\left(y\left(k ; v^{\gamma}, 0\right)-z_{d}+\frac{1}{\sqrt{\gamma}} p^{\gamma}\right) d t d a d x=\int_{Q_{\mathcal{O}}} v q^{\gamma} d t d a d x
$$

and

$$
\frac{1}{\gamma} \int_{Q_{T}} \bar{S}(t, 0, x ; v) S\left(t, 0, x ; v^{\gamma}\right) d t d x=\frac{1}{\sqrt{\gamma}} \int_{Q} \bar{y} p^{\gamma} d t d a d x .
$$

Combining (32), (35) and (36) we obtain that

$$
\int_{Q_{\mathcal{O}}}\left(N v^{\gamma}+q^{\gamma}\right) v d t d a d x=0, \forall v \in L^{2}\left(Q_{\mathcal{O}}\right)
$$

Therefore

$$
v^{\gamma}=-\frac{q^{\gamma}}{N} \text { in } Q_{\mathcal{O}}
$$

Proposition 4 Let $v^{\gamma}=v^{\gamma}(k) \in L^{2}\left(Q_{\mathcal{O}}\right)$ be the solution of (28). Let also $\left(y^{\gamma}, S^{\gamma}, p^{\gamma}, q^{\gamma}\right)$ be the unique solution of (8)-(11). Then there exists a constant 


$$
\begin{aligned}
& C=C(N)>0 \text { independent of } \gamma \text { such that } \\
& \left\|v^{\gamma}\right\|_{L^{2}\left(Q_{\mathcal{O}}\right)} \leq C\left(\left\|z_{d}\right\|_{L^{2}(Q)}+\|k\|_{L^{2}\left(Q_{\omega}\right)}\right), \\
& \left\|y^{\gamma}\right\|_{L^{2}\left(U ; H_{0}^{1}(\Omega)\right)} \leq C\left(\left\|z_{d}\right\|_{L^{2}(Q)}+\|k\|_{L^{2}\left(Q_{\omega}\right)}\right), \\
& \left\|S^{\gamma}\right\|_{L^{2}\left(U ; H_{0}^{1}(\Omega)\right)} \leq C\left(\left\|z_{d}\right\|_{L^{2}(Q)}+\|k\|_{L^{2}\left(Q_{\omega}\right)}\right), \\
& \left\|p^{\gamma}\right\|_{L^{2}\left(U ; H_{0}^{1}(\Omega)\right)} \leq C\left(\left\|z_{d}\right\|_{L^{2}(Q)}+\|k\|_{L^{2}\left(Q_{\omega}\right)}\right), \\
& \frac{1}{\sqrt{\gamma}}\left\|S\left(., 0, . ; v^{\gamma}\right)\right\|_{L^{2}\left(Q_{T}\right)} \leq C\left(\left\|z_{d}\right\|_{L^{2}(Q)}+\|k\|_{L^{2}\left(Q_{\omega}\right)}\right), \\
& \left\|S\left(., 0, . ; v^{\gamma}\right)\right\|_{L^{2}\left(Q_{T}\right)} \leq \sqrt{\gamma} C\left(\left\|z_{d}\right\|_{L^{2}(Q)}+\|k\|_{L^{2}\left(Q_{\omega}\right)}\right) .
\end{aligned}
$$

Proof. It is clear that from (41), we have (42). Since $v^{\gamma}=v^{\gamma}(k) \in L^{2}\left(Q_{\mathcal{O}}\right)$ is the solution of (28), we have that

$$
J^{\gamma}\left(v^{\gamma}\right) \leq J^{\gamma}(v), \forall v \in L^{2}\left(Q_{\mathcal{O}}\right) .
$$

Hence we take $v=-k \chi_{\omega}$ and since $\omega \subset \mathcal{O}$, we obtain

$$
J^{\gamma}\left(v^{\gamma}\right) \leq J^{\gamma}(-k)=N\|k\|_{L^{2}\left(Q_{\omega}\right)}^{2} .
$$

It then follows from the definition of $J^{\gamma}$ given by (27) that

$$
J\left(k ; v^{\gamma}, 0\right)+\frac{1}{\gamma}\|S(., 0, . ; v)\|_{L^{2}\left(Q_{T}\right)}^{2} \leq\left\|z_{d}\right\|_{L^{2}(Q)}^{2}+N\|k\|_{L^{2}\left(Q_{\omega}\right)}^{2},
$$

which in view of (21) implies that

$$
\begin{aligned}
& \left\|y\left(k ; v^{\gamma}, 0\right)\right\|_{L^{2}(Q)} \leq\left\|z_{d}\right\|_{L^{2}(Q)}+\sqrt{N}\|k\|_{L^{2}\left(Q_{\omega}\right)}, \\
& \left\|v^{\gamma}\right\|_{L^{2}\left(Q_{\mathcal{O}}\right)} \leq \frac{1}{\sqrt{N}}\left\|z_{d}\right\|_{L^{2}(Q)}+\|k\|_{L^{2}\left(Q_{\omega}\right)}, \\
& \frac{1}{\sqrt{\gamma}}\left\|S\left(., 0, . ; v^{\gamma}\right)\right\|_{L^{2}\left(Q_{T}\right)} \leq\left\|z_{d}\right\|_{L^{2}(Q)}+\sqrt{N}\|k\|_{L^{2}\left(Q_{\omega}\right)} \text {. }
\end{aligned}
$$

Hence, we obtain from (43b) and (43c), the relations (37) and (41). In view of (37) and (8), we deduce (38). Using (43a) and (9) we obtain (39). From (41) and (10), we deduce (40).

Actually, we can have an estimation of $\frac{1}{\sqrt{\gamma}} p^{\gamma}$ in a an appropriate space. Indeed, , combining (32) and (36), we have that

$$
\begin{aligned}
0 & =\int_{Q} \bar{y}\left\{y\left(k ; v^{\gamma}, 0\right)-z_{d}\right\} d t d a d x+N\left(v^{\gamma}, v\right)_{L^{2}\left(Q_{\mathcal{O}}\right)} \\
& +\frac{1}{\sqrt{\gamma}} \int_{Q} \bar{y} p^{\gamma} d t d a d x, \quad \forall v \in L^{2}\left(Q_{\mathcal{O}}\right) .
\end{aligned}
$$

Consider the following set

$$
\mathcal{E}=\left\{\bar{y}(v), \quad v \in L^{2}\left(Q_{\mathcal{O}}\right)\right\} .
$$


Then $\mathcal{E} \subset L^{2}(Q)$. Define on $\mathcal{E} \times \mathcal{E}$ the inner product:

$$
\begin{aligned}
& \langle\bar{y}(v), \bar{y}(w)\rangle_{\mathcal{E}}=\int_{Q_{\mathcal{O}}} v w d t d a d x+\int_{Q} \bar{y}(v) \bar{y}(w) d t d a d x, \\
& \forall \bar{y}(v), \bar{y}(w) \in \mathcal{E} .
\end{aligned}
$$

Then $\mathcal{E}$ endowed with the norm

$$
\|\bar{y}(v)\|_{\mathcal{E}}^{2}=\|v\|_{L^{2}\left(Q_{\mathcal{O}}\right)}^{2}+\|\bar{y}(v)\|_{L^{2}(Q)}^{2}, \forall \bar{y}(v) \in \mathcal{E}
$$

is an Hilbert space.

We set $T_{\gamma}\left(v^{\gamma}\right)=\frac{1}{\sqrt{\gamma}} p^{\gamma}$. Then in view of $(44)$, we have for any $v \in L^{2}\left(Q_{\mathcal{O}}\right)$,

$$
\int_{Q} T_{\gamma}\left(v^{\gamma}\right) \bar{y}(v) d t d a d x=-\int_{Q} \bar{y}\left\{y\left(k ; v^{\gamma}, 0\right)-z_{d}\right\} d t d a d x-N\left(v^{\gamma}, v\right)_{L^{2}\left(Q_{\mathcal{O}}\right)} .
$$

In view of (37) and (43a), there exists a constant $C=C(N)>0$ independent of $\gamma$ such that

$$
\begin{aligned}
& \left|-\int_{Q} \bar{y}\left\{y\left(k ; v^{\gamma}, 0\right)-z_{d}\right\} d t d a d x-N\left(v^{\gamma}, v\right)_{L^{2}\left(Q_{\mathcal{O}}\right)}\right| \leq \\
& C\left(\left\|z_{d}\right\|_{L^{2}(Q)}+\|k\|_{L^{2}\left(Q_{\omega}\right)}\right)\|\bar{y}(v)\|_{\mathcal{E}} .
\end{aligned}
$$

Hence, we then deduce from (48) and (49) that

$$
\left|\int_{Q} T_{\gamma}\left(v^{\gamma}\right) \bar{y}(v) d t d a d x\right| \leq C\left(\left\|z_{d}\right\|_{L^{2}(Q)}+\|k\|_{L^{2}\left(Q_{\omega}\right)}\right)\|\bar{y}(v)\|_{\mathcal{E}} .
$$

This means that

$$
\left\|T_{\gamma}\left(v^{\gamma}\right)\right\|_{\mathcal{E}^{\prime}}=\left\|\frac{1}{\sqrt{\gamma}} p^{\gamma}\right\|_{\mathcal{E}^{\prime}} \leq C\left(\left\|z_{d}\right\|_{L^{2}(Q)}+\|k\|_{L^{2}\left(Q_{\omega}\right)}\right) .
$$

Remark 5 Note that with estimate obtained in Proposition 4 and (50), we prove the convergence when $\gamma \rightarrow 0$ of the optimality system of Theorem 1, and that, the Low-regret control converges towards the No-regret control which belongs to set $\mathcal{U}$ defined in Remark 3. But No-regret control does not depend linearly on the second control $k$. That is why we study the null controllability of the state equation associated to the Low-regret control $v^{\gamma}$.

\section{Resolution of the problem of controllability}

In this section we are concerned with the Problem 2. More precisely, we consider the null controllability of the state equations associated to the Lowregret control: find $\hat{k}^{\gamma} \in L^{2}\left(Q_{\omega}\right)$ such that if $\hat{y}^{\gamma}=y\left(t, a, x ; \hat{k}^{\gamma} ; \hat{v}^{\gamma}, 0\right), S^{\gamma}=$ $S\left(t, a, x ; \hat{k}^{\gamma} ; \hat{v}^{\gamma}\right), \hat{p}^{\gamma}$ and $\hat{q}^{\gamma}$ are solutions of (8)-(12) then

$$
\hat{y}\left(T, ., . ; \hat{k}^{\gamma} ; \hat{v}^{\gamma}(\hat{k}), 0\right)=0 \text { in } Q_{A} .
$$


To this end we need appropriate Carleman inequalities.

Let us recall that for any nonempty open set $\omega_{0} \subset \omega^{\prime} \subset \omega \subset \subset \Omega$ there exists a function denote $\Psi \in C^{2}(\bar{\Omega})$ such that

$$
\left\{\begin{array}{l}
\Psi(x)=0, \forall x \in \Gamma ; \quad \nabla \Psi(x) \neq 0, \forall x \in \overline{\Omega-\omega_{0}} \\
\Psi(x)>0, \forall x \in \Omega .
\end{array}\right.
$$

We refer to [26] for the existence of such a function $\Psi$. For any $(t, a, x) \in Q$, we set

$$
\begin{aligned}
\eta(t, a, x) & =\frac{e^{2 \lambda\|\Psi\|_{\infty}}-e^{\lambda \Psi(x)}}{t(T-t) a(A-a)}, \\
\varphi(t, a, x) & =\frac{e^{\lambda \Psi(x)}}{t(T-t) a(A-a)} .
\end{aligned}
$$

Let $f \in L^{2}(Q)$ and $z$ be the solution of

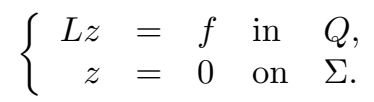

Then we have the following result

Proposition 6 (see [27]) Let $\omega_{0} \subset \omega^{\prime} \subset \omega \subset \subset \Omega$. Let also $\Psi, \eta$ and $\varphi$ be defined as in (52), (53) and (54) respectively. There exist positive constants $\lambda_{0}>1$ and $C=C(\Psi)>0$ such that for $\lambda>\lambda_{0}$ and $s>s_{0}(\lambda)$ such that for all solution of (55) the following inequality holds:

$$
\mathcal{K}(z) \leq C \int_{Q} e^{-2 s \eta}|L z|^{2} d t d a d x+C \int_{0}^{T} \int_{0}^{A} \int_{\omega^{\prime}} s^{3} \lambda^{4} \varphi^{3} e^{-2 s \eta}|z|^{2} d t d a d x
$$

where $s_{0}(\lambda)=C(\Psi) \frac{T A}{4} e^{2 \lambda\|\Psi\|_{\infty}}\left(\frac{T^{2} A^{2}}{4}+T^{2} A^{3}+T^{3} A^{2}+T+A\right)$ and

$$
\mathcal{K}(z)=\int_{Q} e^{-2 s \eta}\left(s \lambda \varphi|\nabla z|+s^{3} \lambda^{4} \varphi^{3}|z|^{2}\right) d t d a d x,
$$

for a suitable function $z$.

Remark 7 By the change of variable $t \mapsto T-t$ and $a \mapsto A-a$, inequality (56) holds also if we replace $L z$ by $L^{*} z$.

We consider the following systems

$$
\left\{\begin{aligned}
L^{*} \rho^{\gamma} & =\psi^{\gamma}+\phi^{\gamma} & & \text { in } \quad Q, \\
\rho^{\gamma} & =0 & & \text { on } \Sigma, \\
\rho^{\gamma}(T, ., .) & =\rho^{T} & & \text { in } Q_{A}, \\
\rho^{\gamma}(., A, .) & =0 & & \text { in } Q_{T},
\end{aligned}\right.
$$




$$
\begin{aligned}
& \left\{\begin{aligned}
L \psi^{\gamma} & =0 & & \text { in } Q, \\
\psi^{\gamma} & =0 & & \text { on } \Sigma, \\
\psi^{\gamma}(0, ., .) & =0 & & \text { in } Q_{A}, \\
\psi^{\gamma}(., 0, .) & =\frac{1}{\sqrt{\gamma}} \zeta^{\gamma}(., 0, .) & & \text { in } Q_{T},
\end{aligned}\right.
\end{aligned}
$$

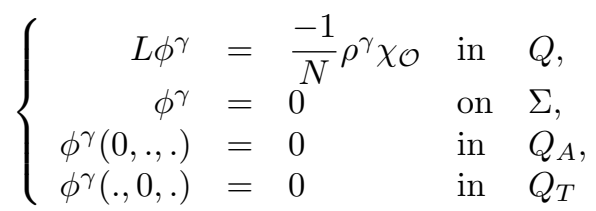

and

$$
\left\{\begin{aligned}
L^{*} \zeta^{\gamma} & =\frac{1}{\sqrt{\gamma}} \phi^{\gamma} & & \text { in } \quad Q, \\
\zeta^{\gamma} & =0 & & \text { on } \Sigma, \\
\zeta^{\gamma}(T, ., .) & =0 & \text { in } & Q_{A}, \\
\zeta^{\gamma}(., A, .) & =0 & & \text { in } Q_{T} .
\end{aligned}\right.
$$

Proposition 8 Under the assumptions of Proposition 6, There exist positive constants $\lambda_{1}$ and $s_{1}$ such that for $\lambda>\lambda_{1}$ and $s>s_{1}$ there exist a constant $C=C(\Psi, N, T, A, s, \lambda)>0$ and a positive weight $\kappa$ such that the following holds true

$$
\begin{aligned}
& \int_{Q} \kappa^{2}\left|\phi^{\gamma}\right|^{2} d t d a d x+\int_{Q} \tilde{\varphi}^{3} e^{-2 s \tilde{\eta}}\left|\rho^{\gamma}\right|^{2} d t d a d x \leq \\
& C(\Psi, N, T, A, s, \lambda) \int_{Q_{\omega}}\left|\rho^{\gamma}\right|^{2} d t d a d x
\end{aligned}
$$

for all solutions of (58)-(61).

Proof. We proceed in two steps.

Step 1. We prove that there exists a positive constant $C$ such that

$$
\int_{Q} \varphi^{3} e^{-2 s \eta}\left|\rho^{\gamma}\right|^{2} d t d a d x \leq C \int_{Q_{\omega}} s^{4} \lambda^{6} \varphi^{7} e^{-2 s \eta}\left|\rho^{\gamma}\right|^{2} d t d a d x .
$$

We consider as in [28], the function $\theta \in C_{0}^{\infty}(\Omega)$ and such that

$$
\begin{aligned}
& 0 \leq \theta \leq 1 \text { on } \omega, \theta=1 \text { on } \omega^{\prime}, \theta=0 \text { on } \Omega \backslash \omega \\
& \frac{\Delta \theta}{\sqrt{\theta}} \in L^{\infty}(\omega), \frac{\nabla \theta}{\sqrt{\theta}} \in\left\{L^{\infty}(\omega)\right\}^{N} .
\end{aligned}
$$

We set $u=s^{3} \lambda^{4} \varphi^{3} e^{-2 s \eta}$. Then it follows from the definition of the functions $\eta$ and $\varphi$ given by (53) and (54) that

$$
\left\{\begin{array}{l}
u(t, 0, x)=u(t, A, x)=0 \\
u(0, a, x)=u(T, a, x)=0
\end{array}\right.
$$

and

$$
\nabla u=u(3 \lambda+2 s \lambda \varphi) \nabla \Psi
$$




$$
\begin{aligned}
\frac{\partial u}{\partial t}+\frac{\partial u}{\partial a}= & u\left[3 \varphi^{-1}\left(\frac{\partial \varphi}{\partial t}+\frac{\partial \varphi}{\partial a}\right)-2 s\left(\frac{\partial \eta}{\partial t}+\frac{\partial \eta}{\partial a}\right)\right], \\
\Delta(u \theta)= & u \theta\left(14 s \lambda^{2} \varphi+4 s^{2} \lambda^{2} \varphi^{2}+9 \lambda^{2}\right)|\nabla \Psi|^{2}+u \Delta \theta \\
& +u \theta(3 \lambda+2 s \lambda \varphi) \Delta \Psi+u(6 \lambda+4 s \lambda \varphi) \nabla \Psi \nabla \theta .
\end{aligned}
$$

If we multiply the first equation in (58) by $\theta u\left(\phi^{\gamma}+\psi^{\gamma}\right)$, where $\phi^{\gamma}$ and $\psi^{\gamma}$ are respectively solution of (60) and (59) and integrate by parts over $Q$, we have

$$
\begin{aligned}
\int_{Q} u \theta\left(\phi^{\gamma}+\psi^{\gamma}\right)^{2} d t d a d x & =\int_{Q} u \theta\left(\phi^{\gamma}+\psi^{\gamma}\right) L^{*} \rho^{\gamma} d t d a d x \\
& =-\frac{1}{N} \int_{Q} \theta u\left(\rho^{\gamma}\right)^{2} \chi_{\mathcal{O}} d t d a d x \\
& +\int_{Q} \theta\left(\phi^{\gamma}+\psi^{\gamma}\right) \rho^{\gamma}\left(\frac{\partial u}{\partial t}+\frac{\partial u}{\partial a}\right) d t d a d x \\
& -\int_{Q}\left(\phi^{\gamma}+\psi^{\gamma}\right) \rho^{\gamma} \Delta(\theta u) d t d a d x \\
& -2 \int_{Q} \rho^{\gamma} \nabla(\theta u) \cdot \nabla\left(\phi^{\gamma}+\psi^{\gamma}\right) d t d a d x \\
& =K_{1}+K_{2}+K_{3}+K_{4},
\end{aligned}
$$

where

$$
\begin{aligned}
K_{1} & =-\frac{1}{N} \int_{Q} \theta u\left(\rho^{\gamma}\right)^{2} \chi_{\mathcal{O}} d t d a d x \\
K_{2} & =\int_{Q} \theta\left(\phi^{\gamma}+\psi^{\gamma}\right) \rho^{\gamma}\left(\frac{\partial u}{\partial t}+\frac{\partial u}{\partial a}\right) d t d a d x \\
K_{3} & =-\int_{Q}\left(\phi^{\gamma}+\psi^{\gamma}\right) \rho^{\gamma} \Delta(\theta u) d t d a d x \\
K_{4} & =-2 \int_{Q} \rho^{\gamma} \nabla(\theta u) \cdot \nabla\left(\phi^{\gamma}+\psi^{\gamma}\right) d t d a d x
\end{aligned}
$$

So,

$$
\begin{aligned}
\int_{0}^{T} \int_{0}^{A} \int_{\omega} u\left(\phi^{\gamma}+\psi^{\gamma}\right)^{2} d t d a d x=K_{1}+K_{2}+K_{3}+K_{4} \\
K_{1} \leq \frac{1}{N} \int_{Q_{\omega}} u\left(\rho^{\gamma}\right)^{2} d t d a d x \\
\quad \leq C(N) \int_{Q_{\omega}} s^{3} \lambda^{4} \varphi^{3} e^{-2 s \eta}\left|\rho^{\gamma}\right|^{2} d t d a d x
\end{aligned}
$$

Using (65), (66) and Young inequality, we obtain that

$$
K_{2} \leq \frac{\delta_{1}}{2} \int_{0}^{T} \int_{0}^{A} \int_{\omega} u\left|\phi^{\gamma}+\psi^{\gamma}\right|^{2} d t d a d x+C(\Psi, A, T) \int_{Q_{\omega}} s^{5} \lambda^{4} \varphi^{7} e^{-2 s \eta}\left|\rho^{\gamma}\right|^{2} d t d a d x
$$




$$
\begin{aligned}
K_{3} & =-\int_{Q}\left(\phi^{\gamma}+\psi^{\gamma}\right) \rho^{\gamma} \Delta(\theta u) d t d a d x \\
& =-\int_{Q} \theta u\left(\phi^{\gamma}+\psi^{\gamma}\right) \rho^{\gamma}\left(14 s \lambda^{2} \varphi+4 s^{2} \lambda^{2} \varphi^{2}+9 \lambda^{2}\right)|\nabla \Psi|^{2} d t d a d x \\
& -\int_{Q} u\left(\phi^{\gamma}+\psi^{\gamma}\right) \rho^{\gamma} \Delta \theta d t d a d x \\
& -\int_{Q} \theta u\left(\phi^{\gamma}+\psi^{\gamma}\right) \rho^{\gamma}(3 \lambda+2 s \lambda \varphi) \Delta \Psi d t d a d x \\
& -2 \int_{Q} u\left(\phi^{\gamma}+\psi^{\gamma}\right) \rho^{\gamma}(3 \lambda+2 s \lambda \varphi) \nabla \Psi . \nabla \theta d t d a d x \\
& =K_{31}+K_{32}+K_{33}+K_{34},
\end{aligned}
$$

where

$$
\begin{aligned}
K_{31}= & -\int_{Q} \theta u\left(\phi^{\gamma}+\psi^{\gamma}\right) \rho^{\gamma}\left(14 s \lambda^{2} \varphi+4 s^{2} \lambda^{2} \varphi^{2}+9 \lambda^{2}\right)|\nabla \Psi|^{2} d t d a d x \\
= & \int_{Q}\left\{\theta^{1 / 2} u^{1 / 2}\left(\phi^{\gamma}+\psi^{\gamma}\right)\right\}\left\{-\theta^{1 / 2} u^{1 / 2} \rho^{\gamma}\left(14 s \lambda^{2} \varphi+4 s^{2} \lambda^{2} \varphi^{2}+9 \lambda^{2}\right)|\nabla \Psi|^{2}\right\} \\
\leq & \frac{\delta_{2}}{2} \int_{0}^{T} \int_{0}^{A} \int_{\omega} u\left|\phi^{\gamma}+\psi^{\gamma}\right|^{2} d t d a d x+C(\Psi) \int_{Q_{\omega}} s^{7} \lambda^{8} \varphi^{7} e^{-2 s \eta}\left|\rho^{\gamma}\right|^{2} d t d a d x \\
& K_{32}=-\int_{Q} u\left(\phi^{\gamma}+\psi^{\gamma}\right) \rho^{\gamma} \Delta \theta d t d a d x \\
& =\int_{Q}\left\{\theta^{1 / 2} u^{1 / 2}\left(\phi^{\gamma}+\psi^{\gamma}\right)\right\}\left\{-u^{1 / 2} \rho^{\gamma} \frac{\Delta \theta}{\theta^{1 / 2}}\right\} d t d a d x \\
\leq & \frac{\delta_{3}}{2} \int_{0}^{T} \int_{0}^{A} \int_{\omega} u\left|\phi^{\gamma}+\psi^{\gamma}\right|^{2} d t d a d x \\
& +C \int_{Q_{\omega}} s^{3} \lambda^{4} \varphi^{3} e^{-2 s \eta}\left|\rho^{\gamma}\right|^{2} d t d a d x \\
K_{33}= & -\int_{Q} \theta u\left(\phi^{\gamma}+\psi^{\gamma}\right) \rho^{\gamma}(3 \lambda+2 s \lambda \varphi) \Delta \Psi d t d a d x \\
= & \int_{Q}\left\{\theta^{1 / 2} u^{1 / 2}\left(\phi^{\gamma}+\psi^{\gamma}\right)\right\}\left\{-\theta^{1 / 2} u^{1 / 2} \rho^{\gamma}(3 \lambda+2 s \lambda \varphi) \Delta \Psi\right\} d t d a d x \\
\leq & \frac{\delta_{4}}{2} \int_{0}^{T} \int_{0}^{A} \int_{\omega} u\left|\phi^{\gamma}+\psi^{\gamma}\right|^{2} d t d a d x \\
+ & C(\Psi) \int_{Q_{\omega}} s^{5} \lambda^{6} \varphi^{5} e^{-2 s \eta}\left|\rho^{\gamma}\right|^{2} d t d a d x
\end{aligned}
$$




$$
\begin{aligned}
K_{34} & =-2 \int_{Q} u\left(\phi^{\gamma}+\psi^{\gamma}\right) \rho^{\gamma}(3 \lambda+2 s \lambda \varphi) \nabla \Psi \cdot \nabla \theta d t d a d x \\
& =\int_{Q}\left\{\theta^{1 / 2} u^{1 / 2}\left(\phi^{\gamma}+\psi^{\gamma}\right)\right\}\left\{-2 u^{1 / 2} \rho^{\gamma}(3 \lambda+2 s \lambda \varphi) \nabla \Psi \cdot \frac{\nabla \theta}{\theta^{1 / 2}}\right\} d t d a d x \\
& \leq \frac{\delta_{5}}{2} \int_{0}^{T} \int_{0}^{A} \int_{\omega} u\left|\phi^{\gamma}+\psi^{\gamma}\right|^{2} d t d a d x \\
& +C(\Psi) \int_{Q_{\omega}} s^{5} \lambda^{6} \varphi^{5} e^{-2 s \eta}\left|\rho^{\gamma}\right|^{2} d t d a d x
\end{aligned}
$$

Therefore

$$
K_{3} \leq \sum_{i=2}^{5} \frac{\delta_{i}}{2} \int_{0}^{T} \int_{0}^{A} \int_{\omega} u\left|\phi^{\gamma}+\psi^{\gamma}\right|^{2} d t d a d x+C(\Psi) \int_{Q_{\omega}} s^{7} \lambda^{8} \varphi^{7} e^{-2 s \eta}\left|\rho^{\gamma}\right|^{2} d t d a d x .
$$

Now we compute the term $K_{4}$. Using (64) and Young inequality, we have

$$
\begin{aligned}
K_{4} & =-2 \int_{Q} \rho^{\gamma} \nabla(\theta u) \cdot \nabla\left(\phi^{\gamma}+\psi^{\gamma}\right) d t d a d x \\
& =-2 \int_{Q} \theta u \rho^{\gamma}(3 \lambda+2 s \lambda \varphi) \nabla \Psi \cdot \nabla\left(\phi^{\gamma}+\psi^{\gamma}\right) d t d a d x-2 \int_{Q} u \rho^{\gamma} \nabla \theta \cdot \nabla\left(\phi^{\gamma}+\psi^{\gamma}\right) d t d a d x \\
& =K_{41}+K_{42},
\end{aligned}
$$

where

$$
\begin{aligned}
K_{41} & =-2 \int_{Q} \theta u \rho^{\gamma}(3 \lambda+2 s \lambda \varphi) \nabla \Psi \cdot \nabla\left(\phi^{\gamma}+\psi^{\gamma}\right) d t d a d x \\
& =\int_{Q}\left\{s^{1 / 2} \varphi^{1 / 2} \theta^{1 / 2} e^{-s \eta} \nabla\left(\phi^{\gamma}+\psi^{\gamma}\right)\right\}\left\{-2 s^{5 / 2} \lambda^{4} \varphi^{5 / 2} \theta^{1 / 2} e^{-s \eta} \rho^{\gamma}(3 \lambda+2 s \lambda \varphi) \nabla \Psi\right\} d t d a d x \\
& \leq \frac{1}{4} \int_{Q_{\omega}} s \varphi e^{-2 s \eta}\left|\nabla\left(\phi^{\gamma}+\psi^{\gamma}\right)\right|^{2} d t d a d x \\
& +C(\Psi) \int_{Q_{\omega}} s^{7} \lambda^{10} \varphi^{7} e^{-2 s \eta}\left|\rho^{\gamma}\right|^{2} d t d a d x
\end{aligned}
$$

and

$$
\begin{aligned}
K_{42} & =-2 \int_{Q} u \rho^{\gamma} \nabla \theta \cdot \nabla\left(\phi^{\gamma}+\psi^{\gamma}\right) d t d a d x \\
& =\int_{Q}\left\{s^{1 / 2} \varphi^{1 / 2} \theta^{1 / 2} e^{-s \eta} \nabla\left(\phi^{\gamma}+\psi^{\gamma}\right)\right\} \cdot\left\{-2 s^{5 / 2} \lambda^{4} \varphi^{5 / 2} e^{-s \eta} \rho^{\gamma} \frac{\nabla \theta}{\theta^{1 / 2}}\right\} d t d a d x \\
& \leq \frac{1}{4} \int_{Q_{\omega}} s \varphi e^{-2 s \eta}\left|\nabla\left(\phi^{\gamma}+\psi^{\gamma}\right)\right|^{2} d t d a d x \\
& +C \int_{Q_{\omega}} s^{5} \lambda^{8} \varphi^{5} e^{-2 s \eta}\left|\rho^{\gamma}\right|^{2} d t d a d x .
\end{aligned}
$$


Thus,

$$
\begin{aligned}
K_{4} & \leq \frac{1}{2} \int_{Q_{\omega}} s \varphi e^{-2 s \eta}\left|\nabla\left(\phi^{\gamma}+\psi^{\gamma}\right)\right|^{2} d t d a d x \\
& +C(\Psi) \int_{Q_{\omega}} s^{7} \lambda^{10} \varphi^{7} e^{-2 s \eta}\left|\rho^{\gamma}\right|^{2} d t d a d x .
\end{aligned}
$$

Finally, in view of (67), we have that

$$
\begin{aligned}
\int_{0}^{T} \int_{0}^{A} \int_{\omega} u\left|\phi^{\gamma}+\psi^{\gamma}\right|^{2} d t d a d x & \leq \sum_{i=1}^{5} \frac{\delta_{i}}{2} \int_{0}^{T} \int_{0}^{A} \int_{\omega} u\left|\phi^{\gamma}+\psi^{\gamma}\right|^{2} d t d a d x \\
& +\frac{1}{2} \int_{Q_{\omega}} s \varphi e^{-2 s \eta}\left|\nabla\left(\phi^{\gamma}+\psi^{\gamma}\right)\right|^{2} d t d a d x \\
& +C(\Psi, N, T, A) \int_{Q_{\omega}} s^{7} \lambda^{10} \varphi^{7} e^{-2 s \eta}\left|\rho^{\gamma}\right|^{2} d t d a d x .
\end{aligned}
$$

Choose in this latter identity $\delta_{i}, 1 \leq i \leq 5$ such that $\sum_{i=1}^{5} \frac{\delta_{i}}{2}=\frac{1}{2}$, then using the fact that $\omega^{\prime} \subset \omega$, we obtain that

$$
\begin{aligned}
& \int_{0}^{T} \int_{0}^{A} \int_{\omega^{\prime}} u\left|\phi^{\gamma}+\psi^{\gamma}\right|^{2} d t d a d x \leq \int_{Q_{\omega}} s \varphi e^{-2 s \eta}\left|\nabla\left(\phi^{\gamma}+\psi^{\gamma}\right)\right|^{2} d t d a d x+ \\
& C(\Psi, N, T, A) \int_{Q_{\omega}} s^{7} \lambda^{10} \varphi^{7} e^{-2 s \eta}\left|\rho^{\gamma}\right|^{2} d t d a d x .
\end{aligned}
$$

Now, applying (56) to $\phi^{\gamma}+\psi^{\gamma}$ where $\phi^{\gamma}$ and $\psi^{\gamma}$ are respectively solution of (60) and (59),

$$
\begin{aligned}
& \int_{Q} e^{-2 s \eta}\left(s \lambda \varphi\left|\nabla\left(\phi^{\gamma}+\psi^{\gamma}\right)\right|+s^{3} \lambda^{4} \varphi^{3}\left|\phi^{\gamma}+\psi^{\gamma}\right|^{2}\right) d t d a d x \leq \\
& C(\Psi) \frac{1}{N^{2}} \int_{Q_{\mathcal{O}}} e^{-2 s \eta}\left|\rho^{\gamma}\right|^{2} d t d a d x+C(\Psi) \int_{0}^{T} \int_{0}^{A} \int_{\omega^{\prime}} s^{3} \lambda^{4} \varphi^{3} e^{-2 s \eta}\left|\phi^{\gamma}+\psi^{\gamma}\right|^{2} d t d a d x,
\end{aligned}
$$

which in view of (68) and the fact that $\varphi^{-1} \in L^{\infty}(Q)$ gives

$$
\begin{aligned}
& \int_{Q} e^{-2 s \eta}\left(s \lambda \varphi\left|\nabla\left(\phi^{\gamma}+\psi^{\gamma}\right)\right|+s^{3} \lambda^{4} \varphi^{3}\left|\phi^{\gamma}+\psi^{\gamma}\right|^{2}\right) d t d a d x \leq \\
& C(\Psi) \int_{Q_{\omega}} s \varphi e^{-2 s \eta}\left|\nabla\left(\phi^{\gamma}+\psi^{\gamma}\right)\right|^{2} d t d a d x+C(\Psi, N) \int_{Q} s^{2} \lambda^{4} e^{-2 s \eta} \varphi^{3}\left|\rho^{\gamma}\right|^{2} d t d a d x+ \\
& C(\Psi, N, T, A) \int_{Q_{\omega}} s^{7} \lambda^{10} \varphi^{7} e^{-2 s \eta}\left|\rho^{\gamma}\right|^{2} d t d a d x .
\end{aligned}
$$

Choosing $\lambda \geq \lambda_{1}=\max \left\{\lambda_{0}, 2 C(\Psi)\right\}$ in (69), we obtain that

$$
\begin{aligned}
& \int_{Q} e^{-2 s \eta}\left(s \lambda \varphi\left|\nabla\left(\phi^{\gamma}+\psi^{\gamma}\right)\right|+s^{3} \lambda^{4} \varphi^{3}\left|\phi^{\gamma}+\psi^{\gamma}\right|^{2}\right) d t d a d x \leq \\
& +C(\Psi, N) \int_{Q} s^{2} \lambda^{4} \varphi^{3} e^{-2 s \eta}\left|\rho^{\gamma}\right|^{2} d t d a d x+ \\
& C(\Psi, N, T, A) \int_{Q_{\omega}} s^{7} \lambda^{10} \varphi^{7} e^{-2 s \eta}\left|\rho^{\gamma}\right|^{2} d t d a d x .
\end{aligned}
$$


Taking into account the Remark 7 and applying (56) to $\rho^{\gamma}$ solution of (58),

$$
\begin{aligned}
& \int_{Q} e^{-2 s \eta}\left(s \lambda \varphi\left|\nabla \rho^{\gamma}\right|+s^{3} \lambda^{4} \varphi^{3}\left|\rho^{\gamma}\right|^{2}\right) d t d a d x \leq \\
& C(\Psi) \int_{Q} e^{-2 s \eta}\left|\phi^{\gamma}+\psi^{\gamma}\right|^{2} d t d a d x+C(\Psi) \int_{Q_{\omega}} s^{3} \lambda^{4} \varphi^{3} e^{-2 s \eta}\left|\rho^{\gamma}\right|^{2} d t d a d x .
\end{aligned}
$$

Using the fact that $\varphi^{-1} \in L^{\infty}(Q)$, we deduce that

$$
\begin{aligned}
& \int_{Q} e^{-2 s \eta}\left(s \lambda \varphi\left|\nabla \rho^{\gamma}\right|+s^{3} \lambda^{4} \varphi^{3}\left|\rho^{\gamma}\right|^{2}\right) d t d a d x \leq \\
& C(\Psi, T, A) s^{2} \lambda^{4} \int_{Q} \varphi^{3} e^{-2 s \eta}\left|\phi^{\gamma}+\psi^{\gamma}\right|^{2} d t d a d x+ \\
& C(\Psi) \int_{Q_{\omega}} s^{3} \lambda^{4} \varphi^{3} e^{-2 s \eta}\left|\rho^{\gamma}\right|^{2} d t d a d x .
\end{aligned}
$$

Combining (70) and (71), then choosing $s \geq s_{1}=\max \left\{s_{0}, 2 C(\Psi, T, A), 2 C(\Psi, N, T, A)\right\}$, we deduce that

$$
\begin{aligned}
& \mathcal{K}\left(\phi^{\gamma}+\psi^{\gamma}\right)+\mathcal{K}\left(\rho^{\gamma}\right) \leq \\
& C(\Psi, N, T, A) \int_{Q_{\omega}} s^{7} \lambda^{10} \varphi^{7} e^{-2 s \eta}\left|\rho^{\gamma}\right|^{2} d t d a d x,
\end{aligned}
$$

which in view of (57) implies that there exists a constant $C=C(\Psi, N, T, A)>0$ such that (63) holds true.

Step 2. We prove that (62).

We set

$$
D=\{(t, a) \in(0, T) \times(0, A) \text { such that } t \geq T / 2 \text { and } a \geq A / 2\} .
$$

Let $\eta$ and $\varphi$ be respectively defined by (53) and (54). For any $x \in \Omega$, we define the functions $\tilde{\eta}$ and $\tilde{\varphi}$ by:

$$
\tilde{\eta}(t, a, x)=\left\{\begin{aligned}
\eta\left(\frac{T}{2}, \frac{A}{2}, x\right) & \text { if }(t, a) \in[(0, T) \times(0, A)] \backslash D, \\
\eta(t, a, x) & \text { if }(t, a) \in D
\end{aligned}\right.
$$

and

$$
\tilde{\varphi}(t, a, x)=\left\{\begin{aligned}
\varphi\left(\frac{T}{2}, \frac{A}{2}, x\right) & \text { if }(t, a) \in[(0, T) \times(0, A)] \backslash D, \\
\varphi(t, a, x) & \text { if }(t, a) \in D .
\end{aligned}\right.
$$

Therefore, replacing respectively $\eta$ and $\varphi$ by $\tilde{\eta}$ and $\tilde{\varphi}$ in (63), we also have

$$
\int_{Q} \tilde{\varphi}^{3} e^{-2 s \tilde{\eta}}\left|\rho^{\gamma}\right|^{2} d t d a d x \leq C(\Psi, N, T, A) \int_{Q_{\omega}} s^{4} \lambda^{6} \varphi^{7} e^{-2 s \tilde{\eta}}\left|\rho^{\gamma}\right|^{2} d t d a d x .
$$

We introduce the function

$$
\hat{\eta}(t, a)=\max _{x \in \Omega} \tilde{\eta}(t, a, x)
$$


and we set

$$
\kappa(t, a)=e^{-s \hat{\eta}(t, a)}
$$

Then $\kappa$ is a positive function of class $\mathcal{C}^{1}$ on $\left[0, T\left[\times\left[0, A\left[\right.\right.\right.\right.$. Moreover, $\frac{\partial}{\partial t} \hat{\eta}(t, a)$ and $\frac{\partial}{\partial a} \hat{\eta}(t, a)$ are positive functions.

So, if we multiply the first equation in (60) by $\kappa^{2} \phi^{\gamma}$ and integrate by part over $\Omega$, we have

$$
\begin{aligned}
& \frac{1}{2} \frac{\partial}{\partial t}\left\|\kappa \phi^{\gamma}\right\|_{L^{2}(\Omega)}^{2}+\frac{1}{2} \frac{\partial}{\partial a}\left\|\kappa \phi^{\gamma}\right\|_{L^{2}(\Omega)}^{2}+\left\|\kappa \nabla \phi^{\gamma}\right\|_{L^{2}(\Omega)}^{2}+r_{0}\left\|\kappa \phi^{\gamma}\right\|_{L^{2}(\Omega)}^{2}= \\
& -\int_{\Omega} \tilde{\mu}_{1} \kappa^{2}\left|\phi^{\gamma}\right|^{2} d x-s \int_{\Omega} \frac{\partial}{\partial t} \hat{\eta} \kappa^{2}\left|\phi^{\gamma}\right|^{2} d x-s \int_{\Omega} \frac{\partial}{\partial a} \hat{\eta} \kappa^{2}\left|\phi^{\gamma}\right|^{2} d x-\frac{1}{N} \int_{\mathcal{O}} \kappa^{2} \rho^{\gamma} \phi^{\gamma} d x,
\end{aligned}
$$

from which we deduce for $r_{0} \geq \frac{1}{2}$ that,

$$
\frac{\partial}{\partial t}\left\|\kappa \phi^{\gamma}\right\|_{L^{2}(\Omega)}^{2}+\frac{\partial}{\partial a}\left\|\kappa \phi^{\gamma}\right\|_{L^{2}(\Omega)}^{2} \leq \frac{1}{N^{2}} \int_{\Omega} \kappa^{2} \rho^{\gamma} d x .
$$

Integrating this latter inequality over $(0, T)$, we obtain

$$
\left\|\kappa(T, a) \phi^{\gamma}(T, a)\right\|_{L^{2}(\Omega)}^{2}+\frac{d}{d a}\left\|\kappa \phi^{\gamma}\right\|_{L^{2}\left(Q_{T}\right)}^{2} \leq \frac{1}{N^{2}}\left\|\kappa \rho^{\gamma}\right\|_{L^{2}\left(Q_{T}\right)}^{2}
$$

because $\phi^{\gamma}(0, a)=0$ in $Q_{A}$. Hence,

$$
\frac{d}{d a}\left\|\kappa \phi^{\gamma}\right\|_{L^{2}\left(Q_{T}\right)}^{2} \leq \frac{1}{N^{2}}\left\|\kappa \rho^{\gamma}\right\|_{L^{2}\left(Q_{T}\right)}^{2} .
$$

This implies that

$$
\left\|\kappa \phi^{\gamma}(., a, .)\right\|_{L^{2}\left(Q_{T}\right)}^{2} \leq \frac{1}{N^{2}}\left\|\kappa \rho^{\gamma}\right\|_{L^{2}(Q)}^{2} \forall a \in(0, A),
$$

because $\phi^{\gamma}(t, 0)=0$ in $Q_{T}$. It then follows from (77) that

$$
\left\|\kappa \phi^{\gamma}\right\|_{L^{2}(Q)}^{2} \leq \frac{A}{N^{2}}\left\|\kappa \rho^{\gamma}\right\|_{L^{2}(Q)}^{2} .
$$

In view of the definition of $\hat{\eta}$ and $\kappa$ given respectively by (75) and (76), we have from (78) that

$$
\int_{Q} \kappa^{2}\left|\phi^{\gamma}\right|^{2} d t d a d x \leq \frac{A}{N^{2}} \int_{Q} \tilde{\varphi}^{3} e^{-2 s \tilde{\eta}}\left|\rho^{\gamma}\right|^{2} d t d a d x
$$

because $\tilde{\varphi}^{-1} \in L^{\infty}(Q)$. Combining this latter inequality with (74), we obtain that

$$
\int_{Q} \kappa^{2}\left|\phi^{\gamma}\right|^{2} d t d a d x \leq C(\Psi, N, T, A) \int_{Q_{\omega}} s^{4} \lambda^{6} \varphi^{7} e^{-2 s \tilde{\eta}}\left|\rho^{\gamma}\right|^{2} d t d a d x
$$


Adding (74) to (79), then using the fact that $\varphi^{7} e^{-2 s \tilde{\eta}} \in L^{\infty}(Q)$, we have (62).

We set

$$
\frac{1}{\varrho^{2}}=\tilde{\varphi}^{3} e^{-2 s \tilde{\eta}} .
$$

Then it follows from (62) that there exists $C=C(\Psi, N, T, A, s, \lambda)>0$, such that for $\left(\rho^{\gamma}, \psi^{\gamma}, \phi^{\gamma}, \zeta^{\gamma}\right)$ verifying systems (58)-(61),

$$
\left\|\kappa \phi^{\gamma}\right\|_{L^{2}(Q)}^{2}+\left\|\frac{1}{\varrho} \rho^{\gamma}\right\|_{L^{2}(Q)}^{2} \leq C \int_{Q_{\omega}}\left|\rho^{\gamma}\right|^{2} d t d a d x
$$

with $\kappa$ defined as in (76).

Multiplying $(8)_{1}$ by $\rho^{\gamma},(9)_{1}$ by $\psi^{\gamma},(11)_{1}$ by $\phi^{\gamma}$ and $(10)_{1}$ by $\zeta^{\gamma}$ and integrating by parts over $Q$, then adding the results, we obtain that

$$
0=\int_{Q_{A}} y^{\gamma}(T, a, x) \rho^{T}(a, x) d a d x-\int_{Q_{\omega}} k \rho^{\gamma} d t d a d x+\int_{Q} z_{d} \phi^{\gamma} d t d a d x .
$$

Thus, null controllability property is equivalent to the problem: find a control $k \in L^{2}\left(Q_{\omega}\right)$ such that for any $\rho^{T} \in L^{2}\left(Q_{A}\right)$, we have

$$
0=\int_{Q_{\omega}} k \rho^{\gamma} d t d a d x-\int_{Q} z_{d} \phi^{\gamma} d t d a d x .
$$

So, to solve this problem we consider for every $\varepsilon>0$, the functional

$$
F_{\varepsilon}\left(\rho^{T}\right)=\frac{1}{2} \int_{Q_{\omega}}\left|\rho^{\gamma}\right|^{2} d t d a d x-\int_{Q} z_{d} \phi^{\gamma} d t d a d x+\varepsilon\left\|\rho^{T}\right\|_{L^{2}\left(Q_{A}\right)}
$$

where $\rho^{\gamma}$ and $\phi^{\gamma}$ verify (58), (59), (60) and (61).

Proposition 9 Let $\varrho$ and $\kappa$ be respectively defined by (80) and (76). Assume that $z_{d} \in L^{2}(Q)$ such that $\frac{1}{\kappa} z_{d} \in L^{2}(Q)$. Then for all $\varepsilon>0$ there exists a unique $\rho_{\varepsilon, \gamma}^{T} \in L^{2}\left(Q_{A}\right)$ such that

$$
F_{\varepsilon}\left(\rho_{\varepsilon, \gamma}^{T}\right)=\inf _{\rho^{T} \in L^{2}\left(Q_{A}\right)} F_{\varepsilon}\left(\rho^{T}\right) .
$$

Moreover, there exists a positive constant $C=C(\Psi, N, T, A, s, \lambda)$ such that, if $\rho_{\varepsilon}^{\gamma}, \psi_{\varepsilon}^{\gamma}, \phi_{\varepsilon}^{\gamma}$ and $\zeta_{\varepsilon}^{\gamma}$ are respectively solutions of (58), (59), (60) and (61) associated to $\rho_{\varepsilon, \gamma}^{T}$ then

$$
\left\|\rho_{\varepsilon}^{\gamma}\right\|_{L^{2}\left(Q_{\omega}\right)} \leq C\left\|\frac{1}{\kappa} z_{d}\right\|_{L^{2}(Q)} .
$$


Proof. It is clear $F_{\varepsilon}$ is strictly convex on $L^{2}\left(Q_{A}\right)$. Using inequalities of energy associated to $\rho_{\varepsilon}^{\gamma}, \psi_{\varepsilon}^{\gamma}, \phi_{\varepsilon}^{\gamma}$ and $\zeta_{\varepsilon}^{\gamma}$, we prove that the functional $F_{\varepsilon}$ is continuous on $L^{2}\left(Q_{A}\right)$. On another hand using Young inequalities, we have

$$
\begin{aligned}
F_{\varepsilon}\left(\rho^{T}\right) & =\frac{1}{2} \int_{Q_{\omega}}\left|\rho^{\gamma}\right|^{2} d t d a d x-\int_{Q} z_{d} \phi^{\gamma} d t d a d x+\varepsilon\left\|\rho^{T}\right\|_{L^{2}\left(Q_{A}\right)} \\
& \geq \frac{1}{2}\left\|\rho^{\gamma}\right\|_{L^{2}\left(Q_{\omega}\right)}^{2}+\varepsilon\left\|\rho^{T}\right\|_{L^{2}\left(Q_{A}\right)}-\frac{\delta}{2}\left\|\frac{1}{\kappa} z_{d}\right\|_{L^{2}(Q)}^{2}-\frac{1}{2 \delta}\left\|\kappa \phi^{\gamma}\right\|_{L^{2}(Q)}^{2},
\end{aligned}
$$

for some $\delta>0$. Using the observability inequality (81), then choosing $\delta=2 C$ with $C=C(\Psi, N, T, A, s, \lambda)$, we deduce that

$$
F_{\varepsilon}\left(\rho^{T}\right) \geq \frac{1}{4} \int_{Q_{\omega}}\left|\rho^{\gamma}\right|^{2} d t d a d x+\varepsilon\left\|\rho^{T}\right\|_{L^{2}\left(Q_{A}\right)}-C\left\|\frac{1}{\kappa} z_{d}\right\|_{L^{2}(Q)}^{2} .
$$

This allowed us to say that $F_{\varepsilon}$ is coercive in $L^{2}\left(Q_{A}\right)$. Consequently, there exists a unique point $\rho_{\varepsilon, \gamma}^{T} \in L^{2}\left(Q_{A}\right)$ where $F_{\varepsilon}$ reaches its minimum.

To characterize the optimal control, we write the Euler-Lagrange optimality conditions:

$$
\lim _{\lambda \rightarrow 0} \frac{F_{\varepsilon}\left(\rho_{\varepsilon, \gamma}^{T}+\lambda \rho^{T}\right)-F_{\varepsilon}\left(\rho_{\varepsilon, \gamma}^{T}\right)}{\lambda}=0, \quad \forall \rho^{T} \in L^{2}\left(Q_{A}\right) .
$$

After some calculations (86) gives

$$
\begin{aligned}
0 & =\int_{Q_{\omega}} \rho_{\varepsilon}^{\gamma} \rho^{\gamma} d t d a d x-\int_{Q} z_{d} \phi^{\gamma} d t d a d x \\
& +\frac{\varepsilon}{\left\|\rho_{\varepsilon, \gamma}^{T}\right\|_{L^{2}\left(Q_{A}\right)}} \int_{Q_{A}} \rho_{\varepsilon, \gamma}^{T} \rho^{T} d a d x, \quad \forall \rho^{T} \in L^{2}\left(Q_{A}\right) .
\end{aligned}
$$

Let $\rho_{\varepsilon, \gamma}^{T}$ be the solution of the problem (84) and $\rho_{\varepsilon}^{\gamma}$ be the solution of (58) associated to $\rho_{\varepsilon, \gamma}^{T}$. Let also $y_{\varepsilon}^{\gamma}, S_{\varepsilon}^{\gamma}, q_{\varepsilon}^{\gamma}$ and $p_{\varepsilon}^{\gamma}$ be the solution associated to $k=k_{\varepsilon}^{\gamma}=\rho_{\varepsilon}^{\gamma} \chi_{\omega}$ of problem (8), (9), (11) and (10) respectively:

$$
\begin{gathered}
\left\{\begin{aligned}
L y_{\varepsilon}^{\gamma}=v_{\varepsilon}^{\gamma} \chi_{\mathcal{O}}+k_{\varepsilon}^{\gamma} \chi_{\omega} & \text { in } Q, \\
y_{\varepsilon}^{\gamma}=0 & \text { on } \Sigma, \\
y_{\varepsilon}^{\gamma}(0, ., .)=0 & \text { in } Q_{A}, \\
y_{\varepsilon}^{\gamma}(., 0, .)=0 & \text { in } Q_{T},
\end{aligned}\right. \\
\left\{\begin{array}{rll}
L^{*} S_{\varepsilon}^{\gamma}=y_{\varepsilon}^{\gamma} & \text { in } Q, \\
S_{\varepsilon}^{\gamma}=0 & \text { on } \Sigma, \\
S_{\varepsilon}^{\gamma}(T, . .)=0 & \text { in } Q_{A}, \\
S_{\varepsilon}^{\gamma}(., A, .)=0 \quad \text { in } Q_{T},
\end{array}\right. \\
\left\{\begin{aligned}
L^{*} q_{\varepsilon}^{\gamma}=y_{\varepsilon}^{\gamma}-z_{d}+\frac{1}{\sqrt{\gamma}} p_{\varepsilon}^{\gamma} & \text { in } Q, \\
q_{\varepsilon}^{\gamma}=0 & \text { on } \Sigma,
\end{aligned}\right. \\
q_{\varepsilon}^{\gamma}(T, . . .)=0 \\
q_{\varepsilon}^{\gamma}(., A, .)=0
\end{gathered}
$$


and

$$
\left\{\begin{aligned}
L p_{\varepsilon}^{\gamma} & =0 & & \text { in } Q, \\
p_{\varepsilon}^{\gamma} & =0 & & \text { on } \Sigma, \\
p_{\varepsilon}^{\gamma}(0, ., .) & =0 & & \text { in } Q_{A}, \\
p_{\varepsilon}^{\gamma}(., 0, .) & =\frac{1}{\sqrt{\gamma}} S_{\varepsilon}^{\gamma}(., 0, .) & & \text { in } Q_{T},
\end{aligned}\right.
$$

with

$$
v_{\varepsilon}^{\gamma}=-\frac{q_{\varepsilon}^{\gamma}}{N} \chi_{\mathcal{O}}
$$

and

$$
k_{\varepsilon}^{\gamma}=\rho_{\varepsilon}^{\gamma} \chi_{\omega} .
$$

Multiplying $(88)_{1}$ by $\rho^{\gamma},(89)_{1}$ by $\psi^{\gamma},(90)_{1}$ by $\phi^{\gamma}$ and $(91)_{1}$ by $\zeta^{\gamma}$ and integrating by parts over $Q$, then adding the results, we have that

$$
0=\int_{Q_{A}} y_{\varepsilon}^{\gamma}(T, a, x) \rho^{T}(a, x) d a d x-\int_{Q_{\omega}} \rho_{\varepsilon}^{\gamma} \rho^{\gamma} d t d a d x+\int_{Q} z_{d} \phi^{\gamma} d t d a d x
$$

which in view of $(87)$ gives

$$
\int_{Q_{A}}\left(y_{\varepsilon}^{\gamma}(T, a, x)+\frac{\varepsilon \rho_{\varepsilon, \gamma}^{T}(a, x)}{\left\|\rho_{\varepsilon, \gamma}^{T}\right\|_{L^{2}\left(Q_{A}\right)}}\right) \rho^{T}(a, x) d a d x=0, \quad \forall \rho^{T} \in L^{2}\left(Q_{A}\right) .
$$

Hence,

$$
y_{\varepsilon}^{\gamma}(T, a, x)=-\frac{\varepsilon \rho_{\varepsilon, \gamma}^{T}(a, x)}{\left\|\rho_{\varepsilon, \gamma}^{T}\right\|_{L^{2}\left(Q_{A}\right)}} .
$$

Consequently,

$$
\left\|y_{\varepsilon}^{\gamma}(T, a, x)\right\|_{L^{2}\left(Q_{A}\right)}=\varepsilon
$$

If we take $\rho^{T}=\rho_{\varepsilon, \gamma}^{T}$ in (87) we obtain that

$$
\begin{aligned}
\left\|\rho_{\varepsilon}^{\gamma}\right\|_{L^{2}\left(Q_{\omega}\right)}^{2} & =\int_{Q} z_{d} \phi^{\gamma} d t d a d x-\varepsilon\left\|\rho_{\varepsilon, \gamma}^{T}\right\|_{L^{2}\left(Q_{A}\right)} \\
& \leq\left\|\frac{1}{\kappa} z_{d}\right\|_{L^{2}(Q)}\left\|\kappa \phi_{\varepsilon}^{\gamma}\right\|_{L^{2}(Q)},
\end{aligned}
$$

which in view of (81) yields

$$
\left\|\rho_{\varepsilon}^{\gamma}\right\|_{L^{2}\left(Q_{\omega}\right)}^{2} \leq C\left\|\frac{1}{\kappa} z_{d}\right\|_{L^{2}(Q)}\left\|\rho_{\varepsilon}^{\gamma}\right\|_{L^{2}\left(Q_{\omega}\right)},
$$

where $C=C(\Psi, N, T, A, s, \lambda)>0$. Thus,

$$
\left\|k_{\varepsilon}^{\gamma}\right\|_{L^{2}\left(Q_{\omega}\right)}=\left\|\rho_{\varepsilon}^{\gamma}\right\|_{L^{2}\left(Q_{\omega}\right)} \leq C\left\|\frac{1}{\kappa} z_{d}\right\|_{L^{2}(Q)},
$$

where $C=C(\Psi, N, T, A, s, \lambda)>0$. This end the proof of Proposition 9 . 


\subsection{Proof of Theorem 2}

We proceed in three steps.

Step 1. We give some a priori estimates on $v_{\varepsilon}^{\gamma}, y_{\varepsilon}^{\gamma}, S_{\varepsilon}^{\gamma}, p_{\varepsilon}^{\gamma}$ and $q_{\varepsilon}^{\gamma}$.

Observing on the one hand that $k_{\varepsilon}^{\gamma}=\rho_{\varepsilon}^{\gamma} \chi_{\omega}$ because of (93), and on the other hand that, $y_{\epsilon}^{\gamma}, S_{\epsilon}^{\gamma}, p_{\epsilon}^{\gamma}$ and $q_{\epsilon}^{\gamma}$ satisfy (88)-(91), using Proposition 4 and the fact that $k_{\varepsilon}^{\gamma}$ satisfies also (85), we deduce that

$$
\begin{aligned}
&\left\|v_{\varepsilon}^{\gamma}\right\|_{L^{2}\left(Q_{\mathcal{O}}\right)} \leq C\left(\left\|z_{d}\right\|_{L^{2}(Q)}+\left\|\frac{1}{\kappa} z_{d}\right\|_{L^{2}(Q)}\right), \\
&\left\|y_{\varepsilon}^{\gamma}\right\|_{L^{2}\left(U ; H_{0}^{1}(\Omega)\right)} \leq C\left(\left\|z_{d}\right\|_{L^{2}(Q)}+\left\|\frac{1}{\kappa} z_{d}\right\|_{L^{2}(Q)}\right), \\
&\left\|S_{\varepsilon}^{\gamma}\right\|_{L^{2}\left(U ; H_{0}^{1}(\Omega)\right)} \leq C\left(\left\|z_{d}\right\|_{L^{2}(Q)}+\left\|\frac{1}{\kappa} z_{d}\right\|_{L^{2}(Q)}\right), \\
&\left\|p_{\varepsilon}^{\gamma}\right\|_{L^{2}\left(U ; H_{0}^{1}(\Omega)\right)} \leq C\left(\left\|z_{d}\right\|_{L^{2}(Q)}+\left\|\frac{1}{\kappa} z_{d}\right\|_{L^{2}(Q)}\right), \\
&\left\|\frac{1}{\sqrt{\gamma}} p_{\varepsilon}^{\gamma}\right\|_{L^{2}(Q)} \leq C_{1}\left(\left\|z_{d}\right\|_{L^{2}(Q)}+\left\|\frac{1}{\kappa} z_{d}\right\|_{L^{2}(Q)}\right), \\
&\left\|q_{\varepsilon}^{\gamma}\right\|_{L^{2}\left(U ; H_{0}^{1}(\Omega)\right.} \leq C_{1}\left(\left\|z_{d}\right\|_{L^{2}(Q)}+\left\|\frac{1}{\kappa} z_{d}\right\|_{L^{2}(Q)}\right), \\
& \frac{1}{\sqrt{\gamma}\left\|S\left(., 0, . ; v_{\varepsilon}^{\gamma}\right)\right\|_{L^{2}\left(Q_{T}\right)} \leq} \quad C\left(\left\|z_{d}\right\|_{L^{2}(Q)}+\left\|\frac{1}{\kappa} z_{d}\right\|_{L^{2}(Q)}\right), \\
&\left\|S\left(., 0, . ; v_{\varepsilon}^{\gamma}\right)\right\|_{L^{2}\left(Q_{T}\right)} \leq \sqrt{\gamma} C\left(\left\|z_{d}\right\|_{L^{2}(Q)}+\left\|\frac{1}{\kappa} z_{d}\right\|_{L^{2}(Q)}\right),
\end{aligned}
$$

where $C=C(\Psi, N, T, A, s, \lambda)>0$ and $C_{1}=C(\gamma, \Psi, N, T, A, s, \lambda)>0$.

Step 2 We study the convergence when $\varepsilon \rightarrow 0$ of the sequences $k_{\varepsilon}^{\gamma}, v_{\varepsilon}^{\gamma}, y_{\varepsilon}^{\gamma}, S_{\varepsilon}^{\gamma}$, $p_{\varepsilon}^{\gamma}$ and $q_{\varepsilon}^{\gamma}$.

In view of (85), (95a)-(95f), we can extract subsequences still denoted by $k_{\varepsilon}^{\gamma}, v_{\varepsilon}^{\gamma}, y_{\varepsilon}^{\gamma}, S_{\varepsilon}^{\gamma}, p_{\varepsilon}^{\gamma}, q_{\varepsilon}^{\gamma}$ such that when $\varepsilon \rightarrow 0$,

$$
\begin{aligned}
& k_{\varepsilon}^{\gamma} \rightarrow \quad \hat{k}^{\gamma} \text { weakly in } L^{2}\left(Q_{\omega}\right), \\
& v_{\varepsilon}^{\gamma} \rightarrow \quad \hat{v}^{\gamma} \text { weakly in } L^{2}\left(Q_{\mathcal{O}}\right), \\
& y_{\varepsilon}^{\gamma} \rightarrow \hat{y}^{\gamma} \text { weakly in } L^{2}\left(U ; H_{0}^{1}(\Omega)\right), \\
& S_{\varepsilon}^{\gamma} \rightarrow \hat{S}^{\gamma} \text { weakly in } L^{2}\left(U ; H_{0}^{1}(\Omega)\right), \\
& q_{\varepsilon}^{\gamma} \rightarrow \hat{q}^{\gamma} \text { weakly in } L^{2}\left(U ; H_{0}^{1}(\Omega)\right), \\
& p_{\varepsilon}^{\gamma} \rightarrow \hat{p}^{\gamma} \text { weakly in } L^{2}\left(U ; H_{0}^{1}(\Omega)\right) .
\end{aligned}
$$


From (92), (96b) and (96e), we obtain

$$
\hat{v}^{\gamma}=-\frac{\hat{q}^{\gamma}}{N} \text { in } Q_{\mathcal{O}}
$$

Multiplying the first equation in (88) by $\xi \in D(Q)$ and integrate by parts over $Q$,

$$
\int_{Q} y_{\varepsilon}^{\gamma} L^{*} \xi d t d a d x=\int_{Q_{\mathcal{O}}} \xi v_{\varepsilon}^{\gamma} d t d a d x+\int_{Q_{\omega}} \xi k_{\varepsilon}^{\gamma} d t d a d x
$$

which passing to the limit when $\varepsilon \rightarrow 0$ gives

$$
\int_{Q} \hat{y}^{\gamma} L^{*} \xi d t d a d x=\int_{Q_{\mathcal{O}}} \xi \hat{v}^{\gamma} d t d a d x+\int_{Q_{\omega}} \xi \hat{k}^{\gamma} d t d a d x
$$

because of (96a), (96b) and (96c). Integrating this latter identity by parts over $Q$,

$$
\int_{Q} \xi L \hat{y}^{\gamma} d t d a d x=\int_{Q_{\mathcal{O}}} \xi \hat{v}^{\gamma} d t d a d x+\int_{Q_{\omega}} \xi \hat{k}^{\gamma} d t d a d x
$$

Hence,

$$
\frac{\partial \hat{y}^{\gamma}}{\partial t}+\frac{\partial \hat{y}^{\gamma}}{\partial a}-\Delta \hat{y}^{\gamma}+\mu \hat{y}^{\gamma}=\hat{v}^{\gamma} \chi_{\mathcal{O}}+\hat{k}^{\gamma} \chi_{\omega} \text { in } Q .
$$

Since $\hat{y}^{\gamma} \in L^{2}\left(U ; H_{0}^{1}(\Omega)\right)$ we have on the one hand that the traces $\hat{y}^{\gamma}(t, a)_{\mid \Gamma}$ exist and belong to $H^{1 / 2}(\Gamma)$ for almost every $(t, a) \in U$, and on the other hand that

$$
\frac{\partial \hat{y}^{\gamma}}{\partial t}+\frac{\partial \hat{y}^{\gamma}}{\partial a}=\left(\Delta \hat{y}^{\gamma}-\mu \hat{y}^{\gamma}+\hat{v}^{\gamma} \chi_{\mathcal{O}}+\hat{k}^{\gamma} \chi_{\omega}\right) \in L^{2}\left(U ; H^{-1}(\Omega)\right) .
$$

Hence we know ( see e.g. [3]) that the traces $\left(\hat{y}^{\gamma}(t, A), \hat{y}^{\gamma}(t, 0)\right)$ exists and belongs to $\left(L^{2}\left(Q_{T}\right)\right)^{2}$ and $\left.\left(\hat{y}^{\gamma}(T, a), \hat{y}^{\gamma}(0, a)\right)\right)$ exists and belongs to $\left(L^{2}\left(Q_{A}\right)\right)^{2}$.

Multiplying the first equation of $(8)$ by $\xi \in \mathcal{C}^{\infty}(\bar{Q})$ such that $\xi=0$ on $\Sigma$, $\xi(T, a)=0$ in $(0, A) \times \Omega, \xi(t, A)=0$ in $(0, T) \times \Omega$, then integrate by parts over $Q$, we get

$$
\int_{Q_{\mathcal{O}}} \xi v_{\varepsilon}^{\gamma} d t d a d x+\int_{Q_{\omega}} \xi k_{\varepsilon}^{\gamma} d t d a d x=\int_{Q} y_{\varepsilon}^{\gamma}\left(-\frac{\partial \xi}{\partial t}-\frac{\partial \xi}{\partial a}-\Delta \xi+\mu \xi\right) d t d a d x .
$$

Passing this latter identity to the limit when $\varepsilon \rightarrow 0$, while taking into account (96a), (96b) and (96c), we get for all $\xi \in \mathcal{C}^{\infty}(\bar{Q})$ such that $\xi=0$ on $\Sigma, \xi(T, a)=$ 0 in $(0, A) \times \Omega, \xi(t, A)=0$ in $(0, T) \times \Omega$,

$$
\int_{Q_{\mathcal{O}}} \xi \hat{v}^{\gamma} d t d a d x+\int_{Q_{\omega}} \xi \hat{k}^{\gamma} d t d a d x=\int_{Q} \hat{y}^{\gamma}\left(-\frac{\partial \xi}{\partial t}-\frac{\partial \xi}{\partial a}-\Delta \xi+\mu \xi\right) d t d a d x
$$


which integrating by parts over $Q$ and using (98) gives for any $\xi \in \mathcal{C}^{\infty}(\bar{Q})$ such that $\xi=$ 0 on $\Sigma, \xi(T, a)=0$ in $(0, A) \times \Omega, \xi(t, A)=0$ in $(0, T) \times \Omega$,

$$
\begin{aligned}
& 0=\int_{Q_{A}} \hat{y}^{\gamma}(0, a, x) \xi(0, a, x) d a d x \\
& +\int_{Q_{T}}^{\hat{y}^{\gamma}(t, 0, x) \xi(t, 0, x) d t d x} \\
& +\int_{\Sigma} \hat{y}^{\gamma} \frac{\partial \xi}{\partial \nu} d t d a d x
\end{aligned}
$$

Taking in (99) successively $\xi(0, a)=0$ in $Q_{A}$ and $\xi(t, 0)=0$ in $Q_{T}$; then $\xi(t, 0)=0$, we successively obtain

$$
\hat{y}^{\gamma}=0 \text { on } \Sigma
$$

and

$$
\hat{y}^{\gamma}(0, a)=0 \text { in } Q_{A} .
$$

Finally, we obtain from (99) that

$$
\hat{y}^{\gamma}(., 0, .)=0 \text { in } Q_{T} .
$$

Thus, from (98), (100), (101) and (102) it follows that $\hat{y}^{\gamma}=y\left(\hat{k}^{\gamma} ; \hat{v}^{\gamma}, 0\right)$ satisfies ( 8). Proceeding as for the proof $y_{\varepsilon}^{\gamma}$ in page 24-25, while using (96a)(96f), (95g) and (95e), we prove that when $\varepsilon \rightarrow 0$, we have that

$$
\begin{aligned}
\frac{1}{\sqrt{\gamma}} S\left(., 0, . ; v_{\varepsilon}^{\gamma}\right) & \rightarrow \frac{1}{\sqrt{\gamma}} S\left(., 0, . ; \hat{v}^{\gamma}\right) \text { weakly in } L^{2}\left(Q_{T}\right), \\
\frac{1}{\sqrt{\gamma}} p_{\varepsilon}^{\gamma} & \rightarrow \frac{1}{\sqrt{\gamma}} \hat{p}^{\gamma} \text { weakly in } L^{2}(Q),
\end{aligned}
$$

and finally that $\hat{S}^{\gamma}=S\left(\hat{v}^{\gamma}\right), \hat{p}^{\gamma}, \hat{q}^{\gamma}$ are solution of (9)-(11). Moreover using (94) we prove that when $\varepsilon \rightarrow 0$,

$$
\hat{y}^{\gamma}(T, ., .)=0 \text { in } Q_{A} .
$$

It then follows that $\left(\hat{k}^{\gamma}, \hat{v}^{\gamma}, \hat{y}^{\gamma}=y\left(\hat{k}^{\gamma} ; \hat{v}^{\gamma}, 0\right), \hat{S}^{\gamma}=S\left(\hat{v}^{\gamma}\right), \hat{p}^{\gamma}, \hat{q}^{\gamma}\right)$ is solution of the null controllability problem (8)-(11), (7).

Step 3 We study the convergence when $\varepsilon \rightarrow 0$ of the sequences $\rho_{\varepsilon}^{\gamma}, \psi_{\varepsilon}^{\gamma}, \phi_{\varepsilon}^{\gamma}$, $\zeta_{\varepsilon}^{\gamma}$.

Observing that $k_{\varepsilon}^{\gamma}=\rho_{\varepsilon}^{\gamma} \chi_{\omega}$, where $\rho_{\varepsilon}^{\gamma}, \psi_{\varepsilon}^{\gamma}, \phi_{\varepsilon}^{\gamma}$ and $\zeta_{\varepsilon}^{\gamma}$ satisfy the following systems:

$$
\left\{\begin{aligned}
L^{*} \rho_{\varepsilon}^{\gamma} & =\psi_{\varepsilon}^{\gamma}+\phi_{\varepsilon}^{\gamma} & & \text { in } \quad Q, \\
\rho_{\varepsilon}^{\gamma} & =0 & & \text { on } \Sigma, \\
\rho_{\varepsilon}^{\gamma}(T, . .) & =\rho^{T} & \text { in } & Q_{A}, \\
\rho_{\varepsilon}^{\gamma}(., A, .) & =0 & \text { in } & Q_{T},
\end{aligned}\right.
$$




$$
\begin{gathered}
\left\{\begin{aligned}
L \psi_{\varepsilon}^{\gamma} & =0 & & \text { in } Q, \\
\psi_{\varepsilon}^{\gamma} & =0 & & \text { on } \Sigma, \\
\psi_{\varepsilon}^{\gamma}(0, . .) & =0 & & \text { in } Q_{A}, \\
\psi_{\varepsilon}^{\gamma}(., 0, .) & =\frac{1}{\sqrt{\gamma}} \zeta_{\varepsilon}^{\gamma}(., 0, .) & & \text { in } Q_{T},
\end{aligned}\right. \\
\left\{\begin{array}{rlrl}
L \phi_{\varepsilon}^{\gamma} & =\frac{-1}{N} \rho_{\varepsilon}^{\gamma} \chi_{\mathcal{O}} & & \text { in } Q, \\
\phi_{\varepsilon}^{\gamma} & =0 & & \text { on } \Sigma, \\
\phi_{\varepsilon}^{\gamma}(0, ., .) & =0 & \text { in } Q_{A}, \\
\phi_{\varepsilon}^{\gamma}(., 0, .) & =0 & & \text { in } Q_{T}
\end{array}\right.
\end{gathered}
$$

and

$$
\left\{\begin{array}{rlrl}
L^{*} \zeta_{\varepsilon}^{\gamma} & =\frac{1}{\sqrt{\gamma}} \phi_{\varepsilon}^{\gamma} & \text { in } & Q, \\
\zeta_{\varepsilon}^{\gamma} & =0 & & \text { on } \Sigma, \\
\zeta_{\varepsilon}^{\gamma}(T, ., .) & =0 & \text { in } & Q_{A}, \\
\zeta_{\varepsilon}^{\gamma}(., A, .) & =0 & \text { in } Q_{T},
\end{array}\right.
$$

it follows from (81) that

$$
\left\|\kappa \phi_{\varepsilon}^{\gamma}\right\|_{L^{2}(Q)}^{2}+\left\|\frac{1}{\varrho} \rho_{\varepsilon}^{\gamma}\right\|_{L^{2}(Q)}^{2} \leq C \int_{Q_{\omega}}\left|\rho_{\varepsilon}^{\gamma}\right|^{2} d t d a d x
$$

which in view of $(85)$ gives

$$
\left\|\kappa \phi_{\varepsilon}^{\gamma}\right\|_{L^{2}(Q)}^{2}+\left\|\frac{1}{\varrho} \rho_{\varepsilon}^{\gamma}\right\|_{L^{2}(Q)}^{2} \leq C\left\|\frac{1}{\kappa} z_{d}\right\|_{L^{2}(Q)}^{2},
$$

where $C=C(\Psi, N, T, A, s, \lambda)>0$.

For any positive function $\Theta \in L^{\infty}(Q)$ and any bounded domain $X$, we set $L^{2}(\Theta, X)=\left\{\varphi \in L^{2}(X), \int_{X} \Theta|\varphi|^{2} d X<\infty\right\}$. Then we have that $L^{2}(X) \subset$ $L^{2}(\Theta, X)$ and the canonical injection is continuous. Thus, in view of (110)

$$
\begin{aligned}
& \phi_{\varepsilon}^{\gamma} \rightarrow \hat{\phi}^{\gamma} \text { weakly in } L^{2}(\kappa, Q), \\
& \rho_{\varepsilon}^{\gamma} \rightarrow \hat{\rho}^{\gamma} \text { weakly in } L^{2}\left(\frac{1}{\varrho}, Q\right),
\end{aligned}
$$

which in view of according to the definitions of $\Psi, \eta, \varphi, \kappa$ and $\varrho$ given by (52), (53), (54), (76) and (80) implies that

$$
\begin{aligned}
& \phi_{\varepsilon}^{\gamma} \rightarrow \hat{\phi}^{\gamma} \text { weakly in } L^{2}(] 0, T-\beta[\times] 0, A-\tau[\times \Omega), \\
& \rho_{\varepsilon}^{\gamma} \rightarrow \hat{\rho}^{\gamma} \text { weakly in } L^{2}(] 0, T-\beta[\times] 0, A-\tau[\times \Omega),
\end{aligned}
$$

where $\beta, \tau>0$. Consequently if we denote by $D(Q)$ the space of infinitely continuously differentiable functions with compact in $Q$ and $D^{\prime}(Q)$ its dual, then

$$
\begin{aligned}
& \phi_{\varepsilon}^{\gamma} \rightarrow \hat{\phi}^{\gamma} \text { weakly in } D^{\prime}(Q), \\
& \rho_{\varepsilon}^{\gamma} \rightarrow \hat{\rho}^{\gamma} \text { weakly in } D^{\prime}(Q)
\end{aligned}
$$


and then,

$$
\begin{aligned}
& \psi_{\varepsilon}^{\gamma} \rightarrow \hat{\psi}^{\gamma} \text { weakly in } D^{\prime}(Q), \\
& \zeta_{\varepsilon}^{\gamma} \rightarrow \hat{\zeta}^{\gamma} \text { weakly in } D^{\prime}(Q) .
\end{aligned}
$$

Therefore, we prove by passing to the limit in systems (106)-(109) that for any $\rho^{T} \in L^{2}\left(Q_{A}\right)$ and $\gamma>0$, the functions $\hat{\rho}^{\gamma}, \hat{\psi}^{\gamma}, \hat{\phi}^{\gamma}$ and $\hat{\zeta}^{\gamma}$ satisfy (15)-(18).

Moreover, using the weak-lower semi-continuity of the norm and (96a), we deduce from (85),

$$
\left\|\hat{k}^{\gamma}\right\|_{L^{2}\left(Q_{\omega}\right)}=\left\|\hat{\rho}^{\gamma}\right\|_{L^{2}\left(Q_{\omega}\right)} \leq C\left\|\frac{1}{\kappa} z_{d}\right\|_{L^{2}(Q)} .
$$

\section{Concluding remarks}

We proposed a method to control hierarchically a population dynamics model with missing birth rate. This method consists in studying a Low-regret control for the follower and the null controllability problem for the leader. The controls found depend on the parameter of the Low-regret control $\gamma$. We can prove that the Low-regret control converges to a control, called No-regret control and even give the characterization of this control. A further work to study the linearity of this control with respect to the follower $k$ so as to consider a Problem 2 with the state associated the No-regret control.

Acknowledgment: The first author was supported by the Alexander von Humboldt foundation, under the programme financed by the BMBF entitled "German research Chairs". The second author is grateful for the facilities provided by the German research Chairs. The third authors was supported by the German Academic Exchange Service (D.A.A.D) under the Scholarship Programme PhD AIMS-Cameroon. The authors would like to express their gratitude to the unknown referees for helpful advice.

\section{References}

[1] B. Ainseba et M. Langlais. Sur un problème de contrôle d'une population structurée en âge et en espace(French) [On a population control problem dynamics with age dependence and spatial structure]. C. R. Acad. Sci. Paris Sér. I Math. 323 (1996), no. 3, 269-274.

[2] M. Langlais. A nonlinear problem in age-dependent population diffusion. SIAM J. Math. Anal. 16 (1985), no. 3, 510-529.

[3] M. G. Garroni, M. Langlais. Age-dependent population diffusion with external constraint. J. Math. Biol. 14 (1982), no. 1, 77-94.

[4] O. Traoré, A. Ouédraogo. Sur un problème de dynamique des populations. (French) [A population dynamics problem]. IMHOTEP J. Afr. Math. Pures Appl. 4 (2003), no. 1, 15-23. 
[5] O. Kavian and O. Traoré. Approximate controllability by birth control for a nonlinear population dynamics model. ESAIM Control Optim. Calc. Var. 17 (2011), no. 4, 1198-1213.

[6] H. von Stackelberg. Markform undGleichgewicht. Springer, Berlin, Germany 1934.

[7] J. L. Lions. Some remarks on Stackelberg's optimization. Math. Models Methods Appl. Sci. 4 (1994), no. 4, 477-487.

[8] O. Nakoulima, Optimal control for distributed systems subject to nullcontrollability. Application to discriminating sentinels. ESAIM Control Optim. Calc. Var. 13 (2007), no. 4, 623-638.

[9] M. Mercan. Optimal control for distributed linear systems subjected to nullcontrollability. Appl. Anal. 92 (2013), no. 9, 1928-1943.

[10] M. Mercan. Optimal control for distributed linear systems subjected to null controllability with constraints on the state. Advances in interdisciplinary mathematical research, 213-232, Springer Proc. Math. Stat., 37, Springer, New York, 2013.

[11] M. Mercan, O. Nakoulima. Control of Stackelberg for a two-stroke problem. Dyn. Contin. Discrete Impuls. Syst. Ser. B Appl. Algorithms 22 (2015), no. 6, 441-463.

[12] M. Kéré, M. Mercan, G. Mophou. Control of Stackelberg for coupled parabolic equations. J. Dyn. Control Syst. 23 (2017), no. 4, 709-733.

[13] B. Jacob, A. Omrane. Optimal control for age-structured population dynamics of incomplete data. J. Math. Anal. Appl.370(1), 42-48 (2010).

[14] J. L. Lions. Contrôle à moindres regrets des systèmes distribués. (French) [Least-regret controls for distributed systems]. C. R. Acad. Sci. Paris Sr. I Math. 315 (1992), no. 12, 1253-1257.

[15] B. Ainseba. Exact and approximate controllability of the age and space population dynamics structured model. J. Math. Anal. Appl. 275 (2002), no. $2,562-574$.

[16] B. Ainseba, S. Anita. Local exact controllability of the age-dependent population dynamics with diffusion. Abstr. Appl. Anal. 6 (2001), no. 6, 357-368.

[17] S. Sawadogo, G. Mophou. Null controllability with constraints on the state for the age-dependent linear population dynamics problem. Adv. Differ. Equ. Control Process. 10 (2012), no. 2, 113-130.

[18] B. Ainseba, M. Langlais. On a population dynamics control problem with age dependence and spatial structure. J. Math. Anal. Appl. 248 (2000), no. 2, 455-474. 
[19] Y. Echarroudi and L. Maniar.Null controllability of a model in population dynamics. Electron. J. Differential Equations 2014, No. 240, 20 pp.

[20] D. Maity, M. Tucsnak, E. Zuazua. Sharp time null controllability of a population dynamics model with age structuring and diffusion. Hal-01764865v1. 2018

[21] O. Traoré. Approximate controllability and application to data assimilation problem for a linear population dynamics model. IAENG Int. J. Appl. Math. 37 (2007), no. 1, Paper 1, 12 pp.

[22] O. Traoré. Null controllability of a nonlinear population dynamics problem. Int. J. Math. Math. Sci. 2006, Art. ID 49279, 20 pp.

[23] M. Mercan, G. M. Mophou. Null controllability with state constraints of a linear backward population dynamics problem. Int. J. Evol. Equ. 9 (2014), no. $1,99120$.

[24] O. Nakoulima, S. Sawadogo. Internal pollution and discriminating sentinel in population dynamics problem. Int. J. Evol. Equ. 2 (2007), no. 1, 29-46.

[25] B. Ainseba, M. Iannelli. Exact controllability of a nonlinear populationdynamics problem. Differential Integral Equations 16 (2003), no. 11, 13691384 .

[26] A. Fursikov, O. Imanuvilov. Controllability of evolution equations. Lecture Notes Series, 34. Seoul National University, Research Institute of Mathematics, Global Analysis Research Center, Seoul, 1996.

[27] O. Traoré. Null controllability and application to data assimilation problem for a linear model of population dynamics. Ann. Math. Blaise Pascal 17 (2010), no. 2, 375-399.

[28] Lus de Teresa. Insensitizing controls for a semilinear heat equation. Comm. Partial Differential Equations 25 (2000), no. 1-2, 39-72.

[29] N. Hegoburu, S. Anita. Null controllability via comparison results for nonlinear age-structured population dynamics. Mathematics of Control, Signals, and Systems (2019) 31:2. https://doi.org/10.1007/s00498-019-0232-x 